University of Wollongong

Research Online

Faculty of Engineering and Information

Faculty of Engineering and Information

Sciences - Papers: Part B

Sciences

2017

\title{
Valleytronics in merging Dirac cones: All-electric-controlled valley filter, valve, and universal reversible logic gate
}

Yee Sin Ang

Singapore University of Technology and Design, ysa190@uowmail.edu.au

Shengyuan Yang

Singapore University of Technology and Design

C Zhang

University of Wollongong, czhang@uow.edu.au

Zhongshui Ma

Peking University, zma@uow.edu.au

Lawrence K. Ang

Singapore University of Technology and Design

Follow this and additional works at: https://ro.uow.edu.au/eispapers1

Part of the Engineering Commons, and the Science and Technology Studies Commons

Research Online is the open access institutional repository for the University of Wollongong. For further information contact the UOW Library: research-pubs@uow.edu.au 


\title{
Valleytronics in merging Dirac cones: All-electric-controlled valley filter, valve, and universal reversible logic gate
}

\author{
Abstract \\ Despite much anticipation of valleytronics as a candidate to replace the aging complementary metal- \\ oxide-semiconductor (CMOS) based information processing, its progress is severely hindered by the lack \\ of practical ways to manipulate valley polarization all electrically in an electrostatic setting. Here, we \\ propose a class of all-electric-controlled valley filter, valve, and logic gate based on the valley-contrasting \\ transport in a merging Dirac cones system. The central mechanism of these devices lies on the \\ pseudospin-assisted quantum tunneling which effectively quenches the transport of one valley when its \\ pseudospin configuration mismatches that of a gate-controlled scattering region. The valley polarization \\ can be abruptly switched into different states and remains stable over semi-infinite gate-voltage windows. \\ Colossal tunneling valley-pseudomagnetoresistance ratio of over $10000 \%$ can be achieved in a valley- \\ valve setup. We further propose a valleytronic-based logic gate capable of covering all 16 types of two- \\ input Boolean logics. Remarkably, the valley degree of freedom can be harnessed to resurrect logical \\ reversibility in two-input universal Boolean gate. The $(2+1)$ polarization states (two distinct valleys plus a \\ null polarization) reestablish one-to-one input-to-output mapping, a crucial requirement for logical \\ reversibility, and significantly reduce the complexity of reversible circuits. Our results suggest that the \\ synergy of valleytronics and digital logics may provide new paradigms for valleytronic-based information \\ processing and reversible computing.

\section{Disciplines} \\ Engineering | Science and Technology Studies

\section{Publication Details} \\ Ang, Y., Yang, S. A., Zhang, C., Ma, Z. \& Ang, L. K. (2017). Valleytronics in merging Dirac cones: All-electric- \\ controlled valley filter, valve, and universal reversible logic gate. Physical Review B: Covering condensed \\ matter and materials physics, 96 (24), 245410-1-245410-13.
}




\title{
Valleytronics in merging Dirac cones: All-electric-controlled valley filter, valve, and universal reversible logic gate
}

\author{
Yee Sin Ang,,$^{1, *}$ Shengyuan A. Yang, ${ }^{1}$ C. Zhang, ${ }^{2}$ Zhongshui Ma, ${ }^{3,4}$ and L. K. Ang ${ }^{1, \dagger}$ \\ ${ }^{1}$ SUTD-MIT International Design Center, Singapore University of Technology and Design, Singapore 487372 \\ ${ }^{2}$ School of Physics, University of Wollongong, NSW 2522, Australia \\ ${ }^{3}$ School of Physics, Peking University, Beijing 100871, China \\ ${ }^{4}$ Collaborative Innovation Center of Quantum Matter, Beijing 100871, China
}

(Received 4 July 2017; revised manuscript received 13 October 2017; published 13 December 2017)

\begin{abstract}
Despite much anticipation of valleytronics as a candidate to replace the aging complementary metal-oxidesemiconductor (CMOS) based information processing, its progress is severely hindered by the lack of practical ways to manipulate valley polarization all electrically in an electrostatic setting. Here, we propose a class of all-electric-controlled valley filter, valve, and logic gate based on the valley-contrasting transport in a merging Dirac cones system. The central mechanism of these devices lies on the pseudospin-assisted quantum tunneling which effectively quenches the transport of one valley when its pseudospin configuration mismatches that of a gate-controlled scattering region. The valley polarization can be abruptly switched into different states and remains stable over semi-infinite gate-voltage windows. Colossal tunneling valley-pseudomagnetoresistance ratio of over $10000 \%$ can be achieved in a valley-valve setup. We further propose a valleytronic-based logic gate capable of covering all 16 types of two-input Boolean logics. Remarkably, the valley degree of freedom can be harnessed to resurrect logical reversibility in two-input universal Boolean gate. The $(2+1)$ polarization states (two distinct valleys plus a null polarization) reestablish one-to-one input-to-output mapping, a crucial requirement for logical reversibility, and significantly reduce the complexity of reversible circuits. Our results suggest that the synergy of valleytronics and digital logics may provide new paradigms for valleytronic-based information processing and reversible computing.
\end{abstract}

DOI: 10.1103/PhysRevB.96.245410

\section{INTRODUCTION}

Valleytronics is an emerging device concept [1-3] based on the manipulation of valley degree of freedom in certain condensed matter systems such as semiconductor quantum well [4], silicon [5], bismuth [6], diamond [7], carbon nanotube [8], graphene [9], Dirac semimetal [10], and transition-metal dichalcogenide (TMD) monolayer [11]. In these materials, electrons can populate multiple low-energy states that are well separated in momentum space, known as valley. The electron's "valley address," or the valley degree of freedom, provides an additional quantum index which can be harnessed for new paradigm of classical and quantum information processing [12]. Valleytronics, alongside with spintronics [13], photonics, and plasmonics [14], has been proposed as a candidate system to replace the aging CMOS technology [15].

Despite recent success in optical manipulation of valley in TMDs $[11,16]$, the experimental progress of generating valley polarization via dc approach remains stagnant due to the lack of practical valley filter, a device that produces valley-polarized current. In general, valley filters can be classified into two types: (i) gauge-field based (GF); and (ii) electrostatic-field based (EF). GF filter [17-31] utilizes an external magnetic field and/or a pseudomagnetic field induced by mechanically straining the crystal [32] to break the valley transport symmetry whereas EF filter mostly relies on energy filtering in properly designed nanostructures [1,33,34] or by forming one-dimensional (1D) topological edge state

\footnotetext{
*Corresponding author: yeesin_ang@sutd.edu.sg

†Corresponding author: ricky_ang@sutd.edu.sg
}

in a domain wall [35-43]. In terms of building compact valleytronic device, EF filter is more advantageous than GF filter as the electrical output of an EF filter is intrinsically compatible with its electric-based controlling knob for valley polarization. This is in contrast to GF filter in which cascading multiple filters would require the formidable tasks of on-chip electricity-to-magnetic or electricity-to-strain conversions to be tamed.

Albeit the practical usefulness of EF valley filter, only a small subset of filters are capable of all-electric control due to the difficulty in breaking valley transport symmetry solely via electrostatic field. Moreover, these filters are severely plagued by stringent conditions such as the need of high-precision structural control of nanostructures or ultralow operating temperature to prevent bulk current from flooding the subtle valley signal carried by $1 \mathrm{D}$ topological edge state $[42,43]$. Thus far, the search for an easy-to-implement, all-electricfield-controlled valley filter remains an ongoing challenge. Beyond valley filters, valley beam splitter, operating via an electron-optics approach [44], has been explored as an alternative building block of valleytronics [45].

In this work, we propose a class of all-electric-controlled valley filtering based on the pseudospin-assisted valleycontrasting quantum tunneling in quasi-two-dimensional system with merging Dirac cones (2MDS) which can be created in a wide class of systems including honeycomb lattice of cold atoms [46], graphene [47,48], few-layer black phosphorus [49-55], Weyl semimetal [56], and antimonene (single layer of antimony [57]). The valley polarization is fully gate controlled and is robust against gate voltage fluctuation. By arranging two filters into valley valve, this pseudospin-assisted filtering effect can produce a colossal valley-pseudomagnetoresistance 
ratio of well over $10000 \%$. This colossal ratio dwarves the tunneling magnetoresistance in conventional magnetic tunnel junctions [58] and the pseudomagnetoresistance in graphene-based pseudospin valve [59], and is on par with the colossal tunneling electroresistance effect in state-of-art ferroelectric tunnel junctions [60]. We further propose a concept of valleytronic logic gates which encompasses all 16 types of two-input Boolean logics.

More remarkably, the valley degree of freedom, which manifests macroscopically in a $(2+1)$ fashion (two valley polarizations plus a null polarization state), can be harnessed as a built-in "valley pigeonhole" for input information storage. This offers a unique possibility of implementing logically reversible universal Boolean gate, a precursor of dissipationless classical reversible computer [61,62], in a valleytronic system. Our results reveal a concrete architecture of valleytronic-based digital information processing. The synergy of valleytronic and Boolean logic may provide a viable new route towards reversible computation which is ultimately required to minimize waste heat generation in classical computer.

\section{A. Concept of pseudospin-assisted valley filter}

The central operating mechanism of the valley filter lies on the pseudospin-assisted valley-contrasting quantum tunneling which effectively quenches the transport of one valley when its pseudospin mismatches that of a gate-controlled scattering region (Fig. 1). In the following, we shall use $2 \mathrm{MDS}$ in few-layer black phosphorus as a model structure to illustrate the valley-filtering effect. It is proposed that the band gap of two-dimensional black phosphorus can be engineered via perpendicular electric field, surface doping, pressure, or laser irradiation [49-55]. Band-gap tuning [53], band-gap closure, and band inversion [49] of few-layer black phosphorus have been realized in recent experiments. The band inversion regime offers a particularly interesting platform for valleytronic applications due to the emergence of two well-separated Dirac cones. In the low-energy regime $\left|\varepsilon_{k}\right|<\Delta_{0}$, where $\Delta_{0}$ is a band-gap parameter, the energy spectrum is composed of two Dirac cones, denoted as $D+$ and $D$ - valleys, with opposite chirality: the pseudospin vector near the Dirac point is locked to the quasiparticle wave vector, and its winding configuration is opposite between the two valleys [Fig. 1(a)]. For $\left|\varepsilon_{k}\right|>\Delta_{0}$, the two valleys merge into a single Fermi surface. In this case, although the valleys are no longer well defined, the pseudospin of forward propagating states still orientates in a fashion that resembles the pseudospin chirality of $D+$ valley for $\varepsilon_{k}>\Delta_{0}$ and of $D-$ valley for $\varepsilon_{k}<-\Delta_{0}$. This creates two well-separated energy windows in which only electrons from a valley of matching chirality are favorably transported due to the conservation of pseudospin [63]. In a device sense, such unusual band topology can be harnessed to construct a valley filter via a "source-channel-drain" transistor setup [Fig. 1(b)]. By gate tuning the Fermi energy $\varepsilon_{F}$ of the channel between the windows of $\varepsilon_{F}>\Delta_{0}, \varepsilon_{F}<-\Delta_{0}$, and $\left|\varepsilon_{F}\right|<\Delta_{0}$, the valley polarizations of the transmitted electrical current can be switched between $D+, D-$ valleys, and null polarization, respectively. Apart from being all-electric controllable, the valley polarization remains remarkably stable over the semi-

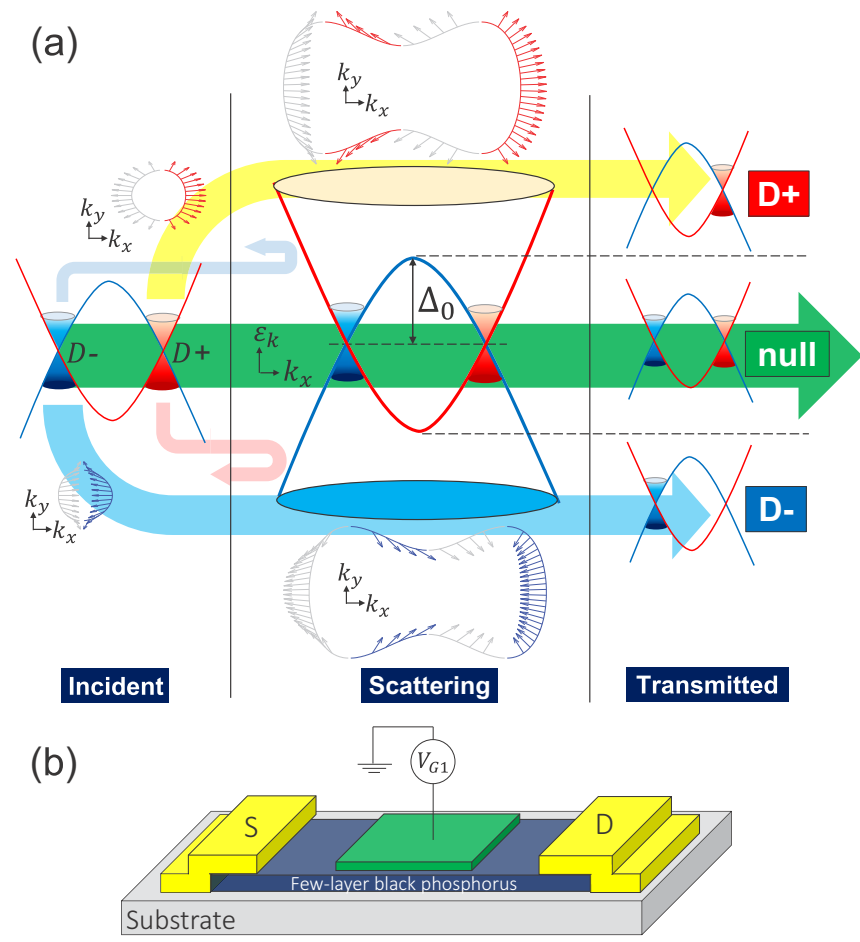

FIG. 1. Concepts of all-electric-controlled valley filter. (a) Band diagram of pseudospin-assisted valley-contrasting quantum tunneling structure in a two-dimensional merging Dirac cone system. The matching and mismatching of pseudospin configuration between the incident and the scattering regions allow the valley polarization of the transmitted current to be all-electrically tuned. The isoenergy contours are shown alongside with the pseudospin vectors of forward (towards-right) propagating states. Backward (towards-left) propagating states are grayed out. (b) Schematic drawing of a valley filter based on a transistor setup.

infinite energy windows of $\varepsilon_{F}>\Delta_{0}$ and $\varepsilon_{F}<-\Delta_{0}$ which can be particularly useful for device applications. The merging transition of $2 \mathrm{MDS}$ in few-layer black phosphorus has been experimentally demonstrated to occur with a sizable energy window of $\approx-200 \mathrm{meV}$ [49]. Such wide energy window may prove a suitable platform for the manipulation of valley in 2MDS provided that the stability and device-fabrication issues of few-layer black phosphorus can be overcome.

\section{B. Concept of reversible valleytronic gate}

The presence of valley degree of freedom adds a new dimension to the Boolean operation in terms of logical reversibility. The reversibility of Boolean logical operation is illustrated in Figs. 2(a)-2(d) by using Boolean loop, a graphical representation of Karnaugh map [64] [see Fig. 1(c)], in which the four vertices represent all $2^{2}$ possible input configurations [the two inputs are represented by $(A, B)$ where $A=0,1$ and $B=0,1]$ and the output state is encoded as follows: empty and filled nodes denote " 0 " and " 1 " output state, respectively. Traditional Boolean gate, such as NAND gate [Fig. 2(a)], is logically irreversible due to the simultaneous presence of multiple filled nodes, i.e., the input is mapped into output via a many-to-one fashion. As the outputs cannot be fully unambiguously reversed back to their corresponding input, 
(a)
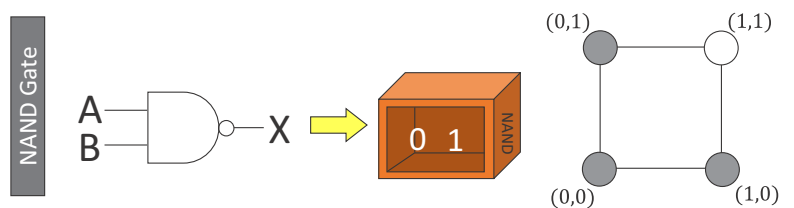

(b)
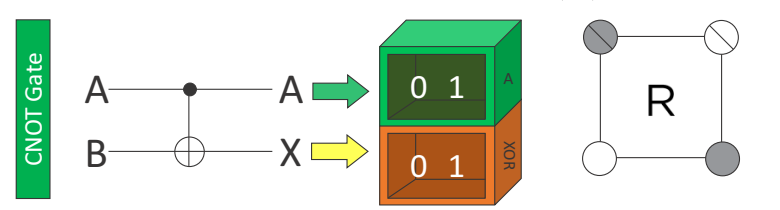

(c)

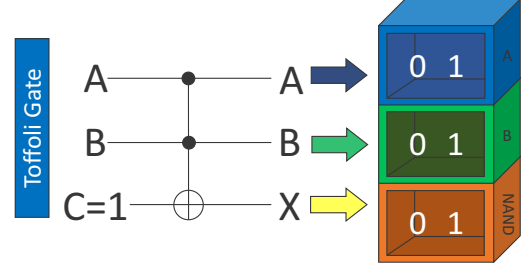

(d)

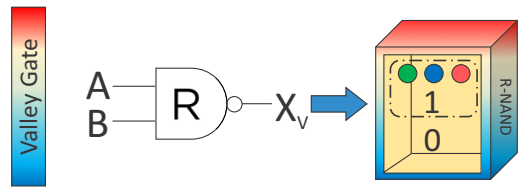

FIG. 2. Reversibility and Boolean loop representations of logic gates. The four vertices of the Boolean loop represents various $(A, B)$ input configuration. Filled (emptied) node denotes "1" ("0") output. Letter "R" emphasizes logical-reversibility. (a) Traditional irreversible NAND. (b) Reversible CNOT. (c) Reversible NAND based on Toffoli gate by holding $C=1$. (d) Valleytronic-based reversible NAND.

part of the input information is inevitably lost. Such logical irreversibility has a profound practical implication: the energy efficiency of Boolean-based computer is ultimately capped at the Landauer's limit, an irreducible waste heat generation of $k_{B} T \ln 2$ per bit of information erased [65].

One potential route to break Landauer's limit is envisaged to be provided by reversible computation which processes information reversibly [61]. Its precursor, universal logic gate, has been actively searched for since early 1970s [66-68]. Controlled-NOT (CNOT) gate, an XOR gate supplemented by an extra output bit identical to one of its input, represents a classic reversible gate [69]. The supplementary bit serves as an information pigeonhole to host two distinct "colors" [denoted by slashed nodes in Fig. 2(b)] that can be used to fully remove the double ambiguity of XOR operation. Universal reversible gate, such as Toffoli gate which provides reversible NAND operations on $(A, B)$ by holding " $C$ " input in " 1 " state, requires two supplementary bits to generate $2^{2}$ distinct colors in order to fully remove the triple ambiguity in the output state. Thus, three out of the $2^{2}$ colors [denoted by filled, slashed, and crossed nodes in Fig. 2(c)] are required to simultaneously preserve both universality and logical reversibility of two-input Boolean gate.

In contrast, the valleytronic-based reversible logic gate proposed in this work operates in a fundamentally different way. The output current produced by the valleytronic system is additionally dressed by $(2+1)$ distinct "valley colors," i.e., two possible states of valley polarizations plus a null

polarization state. These built-in valley colors [denoted, respectively, by red, blue, and green nodes in Fig. 2(d)] can be readily utilized to establish one-to-one mapping between input and output states of a NAND gate. Logical reversibility and universality can thus be simultaneously achieved without the need of adding supplementary bits. More importantly, this allows the valleytronic-based reversible logic gate to retain the simple two-input architecture of conventional Boolean gate and is in stark contrast to the more complicated three-input architecture of Toffoli and Fredkin gates [66,67].

\section{MODEL}

The merging transition of the two Dirac cones in 2MDS can be modeled by an effective Hamiltonian proposed by Montambaux et al. [47]:

$$
\hat{\mathcal{H}}_{\mathbf{K}}=\left(\frac{\hbar^{2} K_{x}^{2}}{2 m^{*}}+\tilde{\Delta}\right) \sigma_{x}+v_{F} K_{y} \sigma_{y},
$$

where $\mathbf{K}=\left(K_{x}, K_{y}\right)$ is the wave vector, $m^{*}$ is the effective mass along $K_{x}$ direction, $v_{F}$ is the Fermi velocity along $K_{y}$ direction, $\sigma=\left(\sigma_{x}, \sigma_{y}\right)$ is the Pauli pseudospin matrix, and $\tilde{\Delta}$ is a band-gap parameter. For simplicity, we transform Eq. (1) into a dimensionless form via the following definitions: $\mathbf{k} \equiv \mathbf{K} / k_{0}$ and $\hat{\mathcal{H}}_{\mathbf{k}} \equiv \hat{\mathcal{H}}_{\mathbf{K}} / \varepsilon_{0}$ where $k_{0}=2 m^{*} v_{F} / \hbar$ and $\varepsilon_{0}=2 m^{*} v_{F}^{2}$ are defined as the characteristic wave vector and energy, respectively. The dimensionless Hamiltonian takes the form of $\hat{\mathcal{H}}_{\mathbf{k}}=\left(k_{x}^{2}+\Delta\right) \sigma_{x}+k_{y} \sigma_{y}$, where $\Delta \equiv \tilde{\Delta} / \varepsilon_{0}$. The energy dispersion is $\varepsilon_{\mathbf{k}}=s \sqrt{\left(k_{x}^{2}+\Delta\right)^{2}+k_{y}^{2}}$ where $s= \pm 1$ denotes conduction and valence band, respectively. Such dispersion exhibits a "semi-Dirac" behavior, i.e., $\varepsilon_{\mathbf{k}}$ along $k_{x}$ axis, and $k_{y}$ axis exhibits nonrelativistic parabolic and ultrarelativistic linear dispersion, respectively [51]. The band topology is crucially determined by the sign of $\Delta$ [Fig. 3(a)]. For $\Delta>0$, the system is a band insulator. The band gap gradually closes as $\Delta \rightarrow 0$. Band inversion, accompanied by the emergence of two Dirac cones situated along the $k_{x}$ axis at $\mathbf{k}=( \pm \sqrt{|\Delta|}, 0)$, occurs for $\Delta<0$. In this case, the Fermi surface is made up of two distinct Dirac pockets of opposite chirality in the low-energy regime of $\left|\varepsilon_{\mathbf{k}}\right|<|\Delta|$ (see Appendix A). The Dirac pockets merge into a single Fermi surface for $\left|\varepsilon_{\mathbf{k}}\right|>|\Delta|$.

We now focus on the $x$-directional transport with $\Delta<0$ where the electron transport exhibits dramatic valley filtering effect. In the presence of a scattering potential $U(x), k_{x}$ is replaced by $k_{x} \rightarrow-i \partial / \partial_{x}$. The eigenstate can be solved from the Schrödinger equation $\hat{\mathcal{H}}_{\mathbf{k}} \psi=\varepsilon_{\mathbf{k}} \psi$ to yield an eigenstate of $\psi^{(\lambda \eta)}\left(\varepsilon_{\mathbf{k}}, k_{y}\right)=\left[1,\left(\lambda \sqrt{\varepsilon_{\mathbf{k}}^{2}-k_{y}^{2}}+i k_{y}\right) / \varepsilon_{\mathbf{k}}\right]^{\mathcal{T}} e^{i k_{x}^{(\lambda \eta)} x}$, where $\mathcal{T}$ denotes transpose, $k_{x}^{(\lambda \eta)}=\lambda \sqrt{\eta\left(\varepsilon_{\mathbf{k}}^{2}-k_{y}^{2}\right)^{1 / 2}-\Delta}, \lambda= \pm 1$ and $\eta= \pm 1$ label the four eigenstates. Figures 3(b) and 3(c) illustrate the pseudospin texture, given by $\mathbf{S}=\left(S_{x}, S_{y}\right)=$ $\psi^{(\lambda \eta)^{\dagger}} \boldsymbol{\sigma} \psi^{(\lambda \eta)}$, along several isoenergy contours with $\varepsilon_{\mathbf{k}}>0$ and $\varepsilon_{\mathbf{k}}<0$, respectively. Two unusual features are observed. First, the $S_{y}$ component $S_{y}=k_{y} / \varepsilon_{\mathbf{k}}$ is identical between the two valleys while the $S_{x}$ component $S_{x}=\left(k_{x}^{(\lambda \eta) 2}+\Delta\right) / \varepsilon_{\mathbf{k}}$ is strongly $k_{x}$ dependent which leads to valley-contrasting transport occurring along the $x$ direction. Second, the two valleys exhibit opposite pseudospin winding configurations and their forward propagating states carry $S_{x}>0$ and $S_{x}<0$, respectively, for $D+$ and $D-$ valleys [denoted by red and 


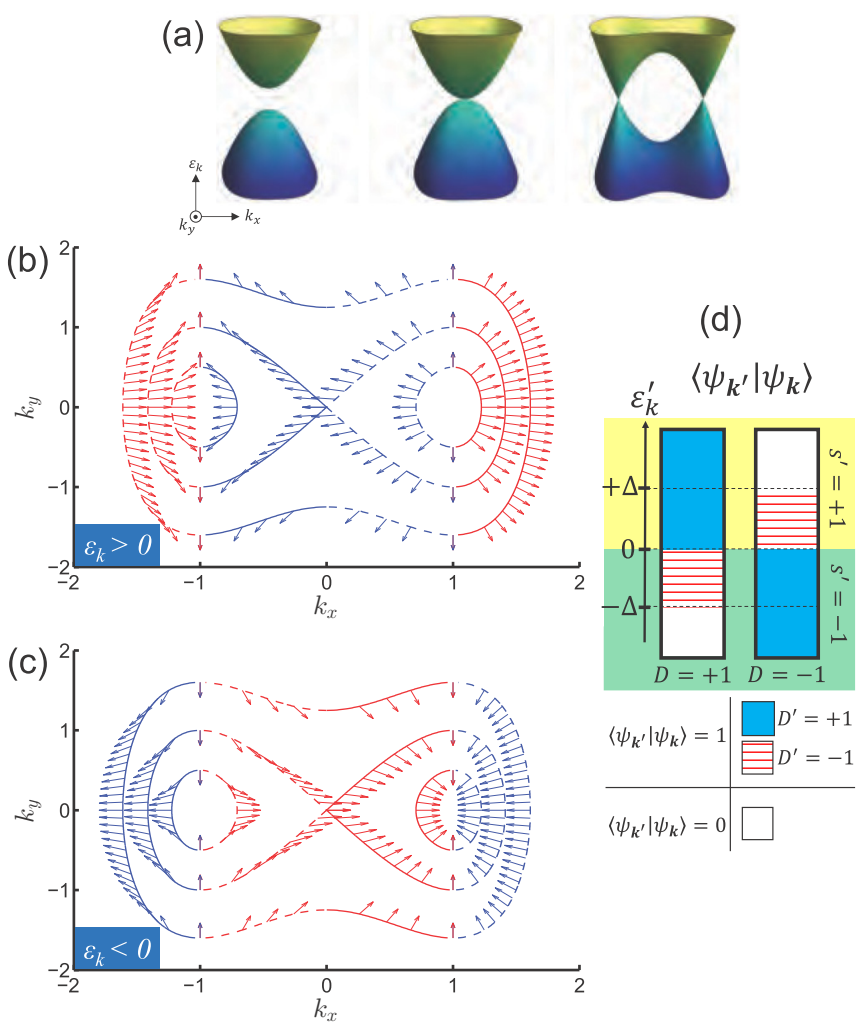

FIG. 3. Band topology, pseudospin configuration, and valleycontrasting transport in merging Dirac cone system. (a) Topological transition of the band structure for $\Delta>0, \Delta=0$, and $\Delta<0$. (b) Pseudospin texture for $\varepsilon_{\mathbf{k}}>0$ and for (c) $\varepsilon_{\mathbf{k}}>0$. Forward and backward propagation is denoted by solid and dashed contour lines, respectively. Blue and red colors denote quasiparticle states with $S_{x}<0$ and $S_{x}>0$, respectively. For (b) and (c), $\Delta=-1$ is used. The innermost, intermediate, and outermost contour lines represent $\varepsilon_{\mathbf{k}}=(0.5,1,1.6)$ and $\varepsilon_{\mathbf{k}}=(-0.5,-1,-1.6)$, respectively, in (b) and (c). (d) Diagram of $\left\langle\psi_{\mathbf{k}^{\prime}, s^{\prime}} \mid \psi_{\mathbf{k}, s^{\prime}}\right\rangle$ for $s=+1$ (left and right bars correspond to $\mathcal{D}^{\prime}= \pm 1$ ). Empty region denotes $\left\langle\psi_{\mathbf{k}^{\prime}, s^{\prime}} \mid \psi_{\mathbf{k}, s^{\prime}}\right\rangle=0$. Filled and horizontally striped regions denote $\left\langle\psi_{\mathbf{k}^{\prime}, s^{\prime}} \mid \psi_{\mathbf{k}, s^{\prime}}\right\rangle=1$ with $\mathcal{D}^{\prime}= \pm 1$, respectively.

blue arrows in Figs. 3(b) and 3(c)]. More importantly, once the two Dirac pockets merge into a single Fermi surface when $\left|\varepsilon_{k}\right|>|\Delta|$, the forward propagation becomes dominated by $S_{x}>0$ for $\varepsilon_{\mathbf{k}}>|\Delta|$ and $S_{x}<0$ for $\varepsilon_{\mathbf{k}}<-|\Delta|$. Thus, the transmission of $D-$ and $D+$ valley states becomes strongly preferred within the semi-infinite energy windows of $\varepsilon_{\mathbf{k}}>|\Delta|$ and $\varepsilon_{\mathbf{k}}<-|\Delta|$, respectively.

This valley-contrasting transport can be further illustrated via a $1 \mathrm{D}$ scattering model (i.e., $k_{y}=0$ ) in which an initial state $\left|\psi_{\mathbf{k}, \mathbf{s}}\right\rangle$ is scattered into a final state $\left|\psi_{\mathbf{k}^{\prime}, s^{\prime}}\right\rangle$ by a pseudospin nonflipping potential $\hat{V}$, as characterized by $\left\langle\psi_{\mathbf{k}^{\prime}, s^{\prime}}|\hat{V}| \psi_{\mathbf{k}, s}\right\rangle=$ $V\left\langle\psi_{\mathbf{k}^{\prime}, s^{\prime}} \mid \psi_{\mathbf{k}, s}\right\rangle$ with $\mathbf{k}^{\prime} \neq \mathbf{k}$ and $V$ is a $\mathbf{k}$-independent potential strength. The transition amplitude can be obtained as $\left\langle\psi_{\mathbf{k}^{\prime}} \mid \psi_{\mathbf{k}}\right\rangle=\left(1+s s^{\prime} \mathcal{D} \mathcal{D}^{\prime}\right) / 2$ where $\mathcal{D}=\operatorname{sign}\left(k_{x}^{2}+\Delta\right)$ and $\mathcal{D}^{\prime}=\operatorname{sign}\left(k_{x}^{\prime 2}+\Delta\right)$ represent the valleys of $\left|\psi_{\mathbf{k}, \mathbf{s}}\right\rangle$ and $\left|\psi_{\mathbf{k}^{\prime}, \mathbf{s}^{\prime}}\right\rangle$, respectively. Note that the valleys are labeled by $\mathcal{D}$ and $\mathcal{D}^{\prime}$ as follows. For $\left(s, s^{\prime}\right)=+1$, the $D \pm$ valley is represented by $\left(\mathcal{D}, \mathcal{D}^{\prime}\right)= \pm 1$. For $\left(s, s^{\prime}\right)=-1$, the $D \mp$ valley is represented by $\left(\mathcal{D}, \mathcal{D}^{\prime}\right)= \pm 1$. For simplicity, we only consider an initial state with $s=1$. For a final state with $\varepsilon_{\mathbf{k}^{\prime}}<|\Delta|, \mathcal{D}^{\prime}$ can be either \pm 1 due to the presence of two distinct Dirac branches. In this case, $\left\langle\psi_{\mathbf{k}^{\prime}} \mid \psi_{\mathbf{k}}\right\rangle=1$ occurs when the condition $s^{\prime} \mathcal{D D}^{\prime}=1$ is fulfilled. Such condition can be translated as follows: the scattering of $\left|\psi_{\mathbf{k}, \mathbf{s}}\right\rangle$ into $\left|\psi_{\mathbf{k}^{\prime}, s^{\prime}}\right\rangle$ is allowed either via intraband $\left(s^{\prime}=+1, \mathcal{D}=\mathcal{D}^{\prime}\right)$ or via interband $\left(s^{\prime}=-1, \mathcal{D}=-\mathcal{D}^{\prime}\right)$ pathway for any valley index (i.e., $\mathcal{D} \pm 1$ ) of the initial state. This corresponds to an "all-pass" scenario where both valleys can simultaneously exhibit unity transition amplitude. In contrast, for a final state with $\varepsilon_{\mathbf{k}^{\prime}}>|\Delta|$, the convergence of two Dirac branches leads to the only possibility of $\mathcal{D}^{\prime}=+1$. This results in a more stringent condition of $s^{\prime} \mathcal{D}=1$ for $\left\langle\psi_{\mathbf{k}^{\prime}, s^{\prime}} \mid \psi_{\mathbf{k}, s}\right\rangle=1$ that corresponds to an exclusively one-valley scattering process of either $\mathcal{D}=+1$ via intraband $\left(s^{\prime}=1\right)$ pathway or $\mathcal{D}=-1$ via interband $\left(s^{\prime}=-1\right)$ pathway. Various possibilities of $\left\langle\psi_{\mathbf{k}^{\prime}, s^{\prime}} \mid \psi_{\mathbf{k}, s^{\prime}}\right\rangle$ are summarized in Fig. 3(d). Here, the quasiparticle scattering can be valley-selectively controlled by switching the final state band index $s^{\prime}$. This valley-selective scattering effect forms the central operating mechanism of the valleytronic devices proposed in this work.

\section{VALLEYTRONIC TRIO: FILTER, VALVE, AND REVERSIBLE LOGIC GATE}

In this section, we show that the highly nontrivial band topology and the pseudospin texture in 2MDS can be harnessed to create a trio of all-electric valleytronic devices: valley filter, valve, and reversible logic gate (see Appendices $\mathrm{B}$ and $\mathrm{C}$ for details of device modeling). In the following, the device modeling is performed using band structure parameters of fewlayer black phosphorus with merging Dirac cones calculated from first principles by Baik et al. [50], i.e., $\varepsilon_{0} \approx 1.3 \mathrm{eV}$ and $k_{0} \approx 1.1 \mathrm{~nm}^{-1}$ [70]. We model the valleytronic devices using a Landauer's ballistic transport formalism [71] for two-dimensional (2D) nanostructures. The ballistic transport picture has been widely used in the modeling of valleyfiltering effect in nanostructures [1,17-29,31,34]. In realistic device, the inevitable presence of impurities, defects, and many-body effects can quantitatively change the results, but the valley-filtering effect shall qualitatively remain robust as recently demonstrated for the case of strained graphene [30].

\section{A. Chiral valley-filtering effect}

As a proof of concept, we first demonstrate the pseudospinassisted valley-selective quantum tunneling, with band diagram shown in Fig. 4(a), by calculating the same-valley transmission probability $T_{ \pm}^{( \pm)}$as a function of incident energy $\varepsilon_{\mathbf{k}}$ and gate voltage $V_{g}$ [Figs. 4(b) and 4(c)]. The $\mathcal{D} \rightarrow \mathcal{D}^{\prime}$ transmission probabilities are denoted as $T_{\mathcal{D}^{\prime}}^{(\mathcal{D})}$ where $\mathcal{D}, \mathcal{D}^{\prime}=$ \pm 1 represents different valley states. The intervalley scattering effect is intrinsically included in this model. Its scattering probabilities $T_{\mp}^{( \pm)}$are strongly suppressed and remain negligibly small for all $V_{g}$ (see Appendix B). For intravalley scattering $T_{ \pm}^{( \pm)}$, a potential barrier $\left(V_{g}>0\right)$ is nearly opaque for $D+$ electrons [Fig. 4(b)] but highly transparent for $D-$ electrons [Fig. 4(c)]. In contrast, the valley preference of 

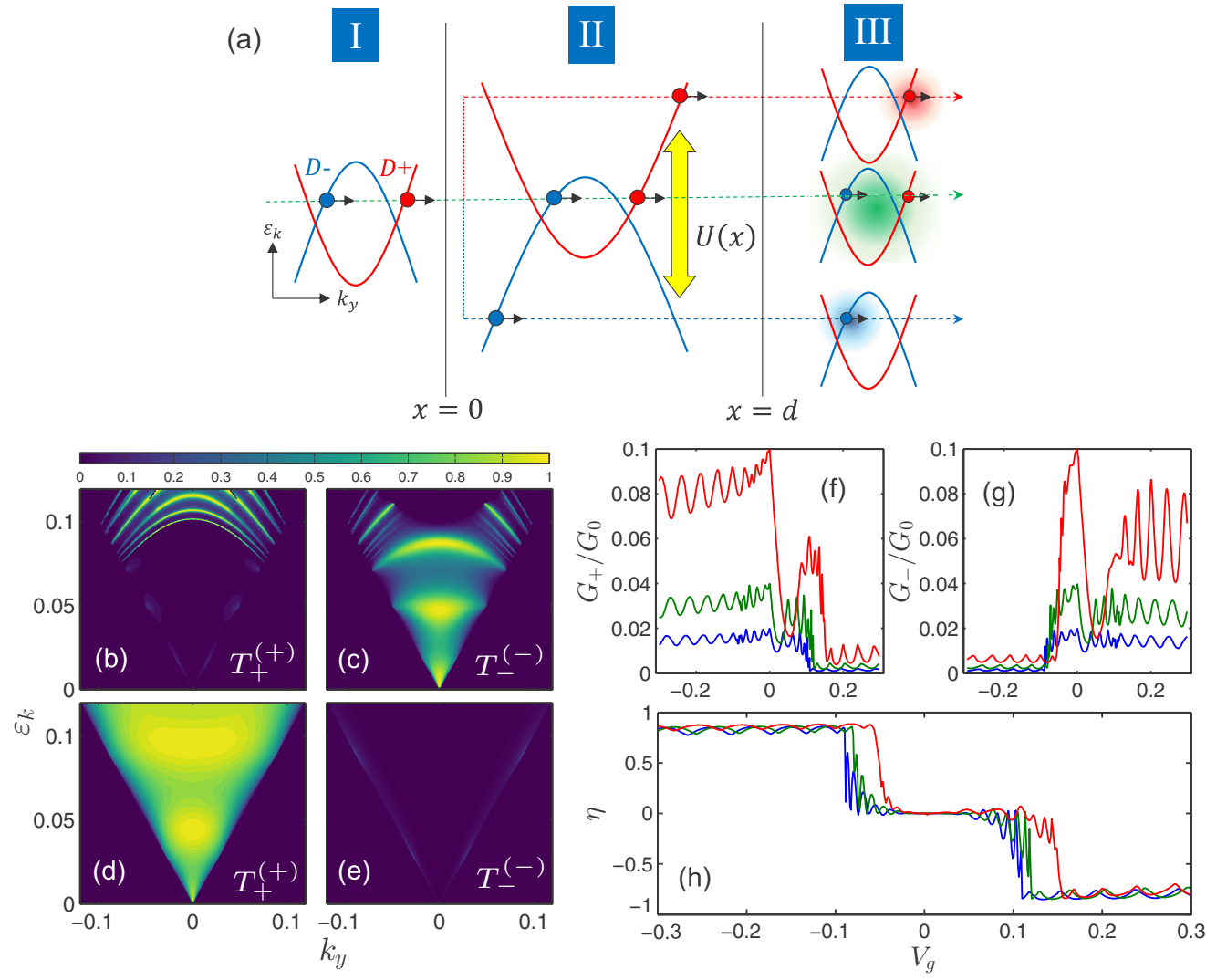

FIG. 4. Operation of valley filter. (a) Band diagram of the valley filter. $\Delta$ is constant throughout regimes I, II, and III. The band alignment of regime II is gate tunable via $U(0<x<d)$. Transmission probabilities $T_{+}^{(+)}$for (b) potential barrier and for (d) potential well. (c), (e) Same as (b) and (d) for $T_{-}^{(-)}$. Valley-polarized conductance in (f) $D+$ and (g) $D-$ channels. Blue, green, and red curves correspond to $\varepsilon_{F} /|\Delta|=(0.1,0.2,0.5)$, respectively. (h) Valley polarization efficiency $\eta . \Delta=-0.1$ and $d=75$ are used.

a potential well $\left(V_{g}<0\right)$ behaves in an opposite fashion: transmission of electrons in $D+$ valley is preferred [Fig. 4(d)] while that of $D$ - valley is strongly suppressed [Fig. 4(e)]. This valley-selective transport is a direct consequence of the matching and mismatching of the pseudospin configurations as discussed above [Fig. 1(a)] and immediately suggests that the proposed device can be operated as a gate-tunable valley filter.

Macroscopically, such valley-selective quantum tunneling effect manifests in the transport measurement by exhibiting valley-polarized electrical conductance. To illustrate this, we separate the contribution from $D \pm$ valleys by defining a valley-dependent electrical conductance as $G_{ \pm}\left(\varepsilon_{F}, V_{g}\right)=$ $G_{0} \sum_{\mathcal{D}^{\prime}= \pm} \int d k_{y} T_{ \pm}^{\left(\mathcal{D}^{\prime}\right)}\left(k_{y}, \varepsilon_{F}, V_{g}\right)$ where $\varepsilon_{F}$ is the Fermi level of the sample, $G_{0} \equiv W g_{0} k_{0} / 2 \pi, g_{0} \equiv 4 e^{2} / h$, and $W$ is the sample width. The $V_{g}$ dependence of $G_{ \pm}$is shown in Figs. 4(f) and 4(g). Apart from the expected conductance oscillations due to Fabry-Pérot interference, it can be seen that $G_{ \pm}$dominates well-separated regime of $V_{g}$. For $V_{g}<0$ and $V_{g}>0$, electrical conduction occurs almost exclusively via $G_{+}$and $G_{-}$, respectively, thus demonstrating a gate-tunable valley polarization of the electrical current. Only at the vicinity of $V_{g}=0, G_{+}$and $G_{-}$mixes due to the presence of both $D \pm$ transmission pathway. The sharp dip of $G_{ \pm}$when $V_{g} \approx \varepsilon_{F}$ corresponds to the case when $\varepsilon_{F}$ is situated at the Dirac point which has a vanishing density of states.
The valley-filtering effect can be characterized by the valley polarization efficiency

$$
\eta\left(\varepsilon_{F}, V_{g}\right)=\frac{G_{+}\left(\varepsilon_{F}, V_{g}\right)-G_{-}\left(\varepsilon_{F}, V_{g}\right)}{G_{+}\left(\varepsilon_{F}, V_{g}\right)+G_{-}\left(\varepsilon_{F}, V_{g}\right)},
$$

which exhibits two remarkable behaviors [Fig. 4(h)]. First, high degree of valley polarization persists over a semi-infinite $V_{g}$ window, indicating high robustness against noise fluctuations of $V_{g}$. Second, the valley polarization can be switched "off" into a stable null-polarization state by setting the gate voltage to $-\left(|\Delta|-\varepsilon_{F}\right) \lesssim V_{g} \lesssim\left(|\Delta|+\varepsilon_{F}\right)$. Together with the two $D \pm$ polarization states, this forms $(2+1)$ stable valley states which can be used to implement universal reversible Boolean logics as shown below.

\section{B. Valley valve and colossal valley pseudomagnetoresistance}

We now propose a valley valve capable of performing current on-off switching. The valley valve is composed of two gates $V_{G 1}$ and $V_{G 2}$ that serve as the functional core of the valve, and a third "selector" gate $V_{s}$ which, in analogy to the role of "analyzer" in an optical polarizer-analyzer system, switches the valley valve into $D+$ and $D$ - modes by providing an additional stage of filtering [Fig. 5(a)].

The tuning of $V_{G 1}$ and $V_{G 2}$ creates four quadrants of parallel and antiparallel configurations as denoted by $\mathrm{Q} 1$ to Q4 in Fig. 5(b). The $D+$ and $D-$ conduction dominates Q3 


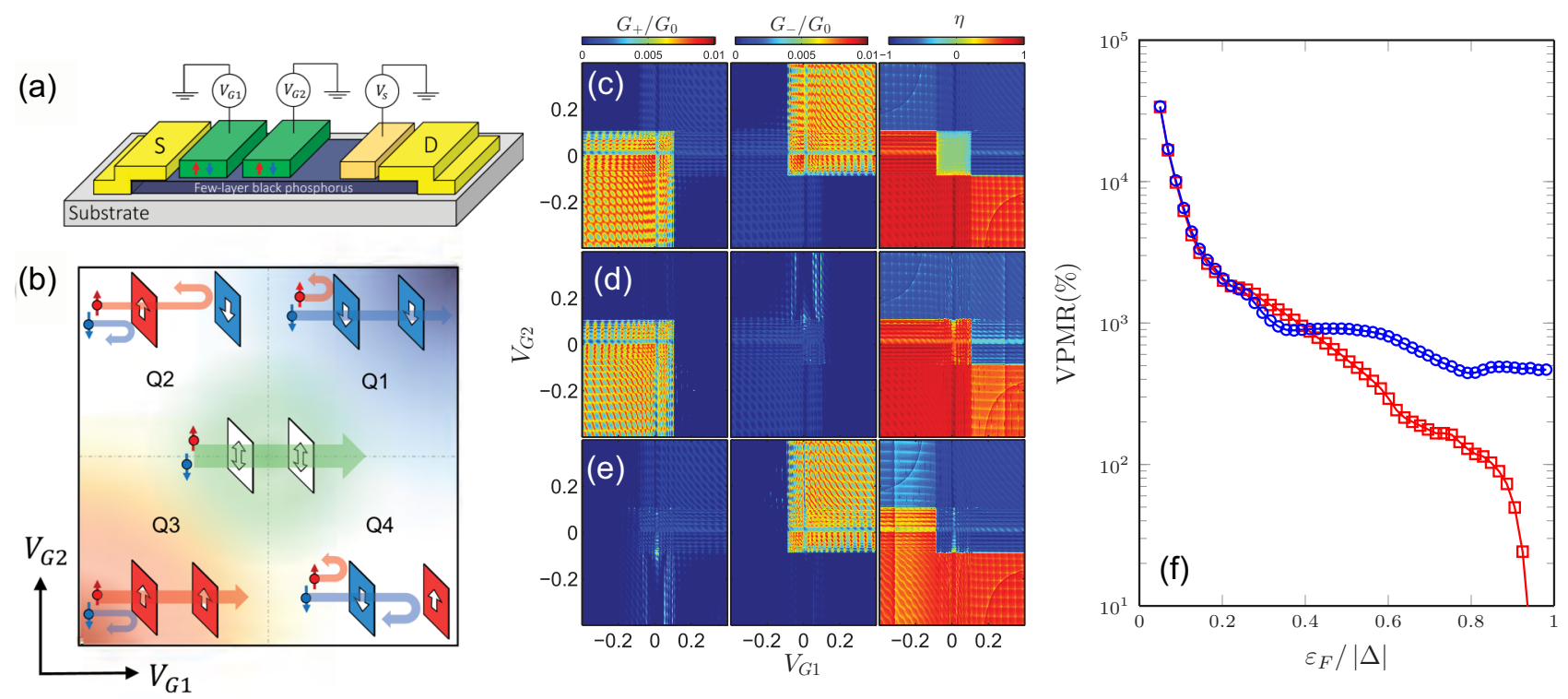

FIG. 5. Operation of valley valve and colossal valley-pseudomagnetoresistance effect. (a) Schematic drawing of valley valve. (b) Parallel and antiparallel configurations and their respective quadrant in the $V_{G 1}-V_{G 2}$ space. (c) Valley-dependent conductance $G_{ \pm}$and the corresponding $\eta$. (d), (e) Same as (c) but operated in $D+$ and $D-$ modes, respectively, via $V_{s}=-0.2$ and 0.2 . (f) VPMR ratio of Q1 $\leftrightarrows$ Q2 (denoted by red squares) and Q3 $\leftrightarrows \mathrm{Q} 2$ (denoted by blue circles) is shown. The VPMR ratios of Q1 $\leftrightarrows$ Q4 and Q3 $\leftrightarrows$ Q4 are approximately identical to this plot and hence are not shown. The following parameters are used: $\Delta=-0.1, \varepsilon_{F} /|\Delta|=0.1$, the barrier width corresponding to $V_{G 1,2, s}$ is set to $d_{\text {gate }}=75$ and are separated by $d_{\text {inter }}=20$.

and Q1, respectively, and the current can be switched off by operating the valve in $\mathrm{Q} 2$ and $\mathrm{Q} 4$. Figures 5(c)-5(e) show the numerical results of $G_{ \pm}\left(\varepsilon_{F}, V_{G 1}, V_{G 2}\right)$ and the corresponding $\eta$. The conductances exhibit Fabry-Pérot oscillations due to interference of wave function. The $G_{+}$and $G_{-}$plateaus occur in a semi-infinite regime defined by $V_{g 1, g 2}<\left(|\Delta|+\varepsilon_{F}\right)$ and $V_{G 1, G 2}>-\left(|\Delta|-\varepsilon_{F}\right)$, respectively. The intersections of $G_{+}$ and $G_{-}$plateaus form a central null-polarization "square" (i.e., $\eta \approx 0)$ as bounded by $-\left(|\Delta|-\varepsilon_{F}\right)<V_{G 1, G 2}<\left(|\Delta|+\varepsilon_{F}\right)$. A valley valve operating in $D+$ mode can be obtained by switching $V_{G 2}$ between $\mathrm{Q} 2 \leftrightarrows \mathrm{Q} 3$ while fixing $V_{G 1}$ at a negative value, or by switching $V_{G 1}$ between $\mathrm{Q} 3 \leftrightarrows \mathrm{Q} 4$ while fixing $V_{G 2}$ at a negative value. Similarly, a $D-$ mode valley valve can be obtained via the switching of $V_{G 1}\left(V_{G 2}\right)$ with Q1 $\leftrightarrows$ Q2 while fixing $V_{G 2}$ at a positive value, or equivalently via the switching of $V_{G 2}$ with Q1 $\leftrightarrows$ Q4 while fixing $V_{G 1}$ at a positive value. In Figs. 5(d) and 5(e), the $D-$ and $D+$ conduction blocks are selectively suppressed by setting $V_{s}=-0.2$ and 0.2 , respectively. This switches the valley valve into an exclusively $D+$ or $D-$ mode.

The valley valve can be characterized by a tunneling valley-pseudomagnetoresistance (VPMR) ratio, analogous to the tunneling magnetoresistance (TMR) in magnetic tunnel junction, which is defined as

$$
\mathrm{VPMR}=\frac{\bar{G}_{\mathrm{total}}^{P}-\bar{G}_{\mathrm{total}}^{A P}}{\bar{G}_{\mathrm{total}}^{A P}},
$$

where $\bar{G}_{\text {total }}^{P}$ and $\bar{G}_{\text {total }}^{A P}$ represent the total conductance averaged over a range of $V_{G 1,2}$ in parallel and antiparallel configurations. The VPMR for $D-$ conductance block on-off switching, i.e., $\mathrm{Q} 1 \leftrightarrows \mathrm{Q} 2$, that of the $D+$ conductance block, i.e., Q3 $\leftrightarrows \mathrm{Q} 2$, is shown in Fig. 5(f) with $V_{s}=0$. For $\varepsilon_{F} \rightarrow|\Delta|$, the VPMR of Q3 $\leftrightarrows$ Q2 gradually stabilizes at $\sim 400 \%$ while the VPMR of Q1 $\leftrightarrows \mathrm{Q} 2$ is severely degraded due to the disappearance of $D$ - conductance block when the two Dirac cones merge. Remarkably, the VPMR exhibits a colossal value of well over $10000 \%$ at small $\varepsilon_{F}$. This value greatly exceeds the pseudomagnetoresistance (PMR) of $\sim 100 \%$ in graphenebased pseudospin valve [59] and TMR 1000\% in traditional magnetic tunnel junction [58], and is on par with state-of-art tunneling electroresistance (TER) of up to TER $10000 \%$ in ferroelectric tunnel junctions [60]. This colossal VPMR originates from the pseudospin-assisted tunneling described above, which effectively quenches the conduction current when there is a mismatch of pseudospin.

\section{Universal reversible valleytronic logic gate}

We now show that the existence of $D \pm$ blocks and the central null-polarization square in the conductance spectrum in Figs. 5(c)-5(e) allows the valley valve device to be operated as a two-input Boolean combinational logic gate. We first translate this conductance spectrum into a simplified valleytransport phase diagram (VPD) [Figs. 6(a)-6(c)]. In Fig. 6(a), selector voltage is set to $V_{s}=0$, i.e., both $D \pm$ channels are opened. In this case, both the $D+$ (red) and the $D-$ (blue) blocks of conductance plateau are present and their intersection forms a central green block of null polarization. The VPDs in Figs. 6(b) and 6(c) correspond the case of $D+$ and $D$ - modes by, respectively, setting $V_{s}=-0.2$ and 0.2 .

To demonstrate the logical operation of the proposed valleytronic gate, we employ a graphical Boolean loop analysis based on the Karnaugh's map approach [64]. Such method provides a simplified tool as it directly maps abstract Boolean logical operations onto the conductance spectrum of a physical 
(a)

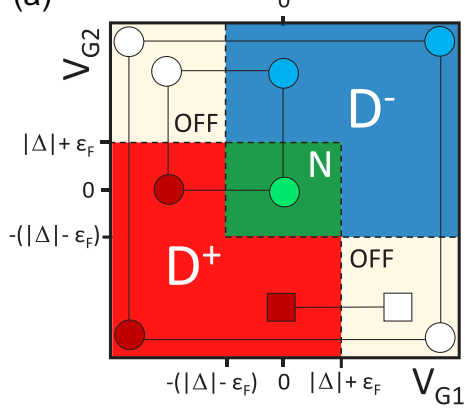

(b)

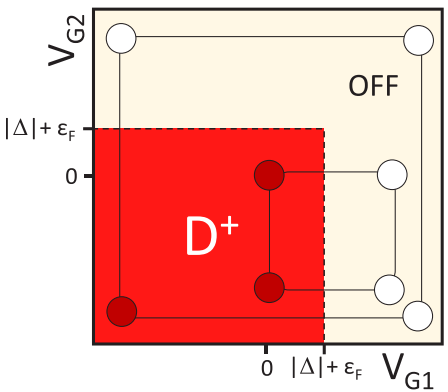

(c)

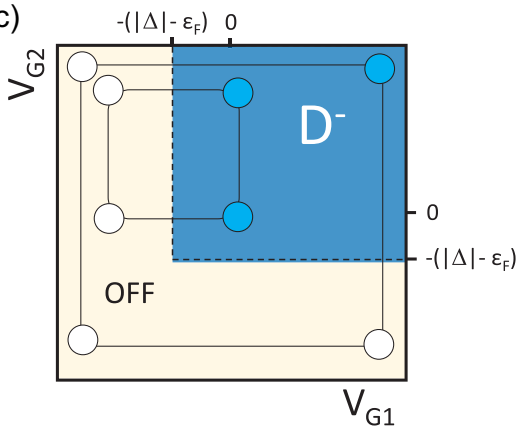

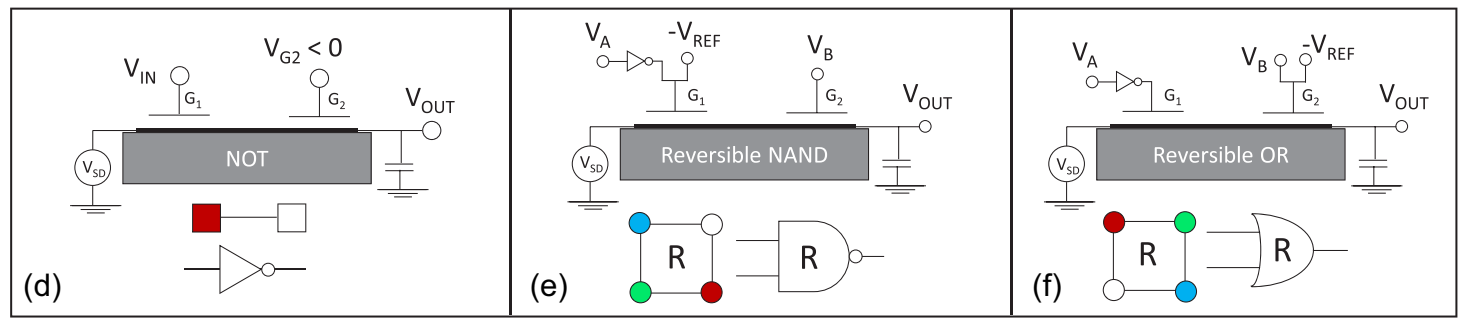

FIG. 6. Valley-transport phase diagrams (VPD) and universal reversible valleytronic logic gates. (a) VPD of the valley filter. (b) VPD for $D+$ mode and (c) $D-$ mode. One-input NOT operation is indicated by a single Boolean line in (a). The larger and the smaller loops in (a) represent Class-2A and Class-3 operations, respectively. In (b) and (c), the larger and smaller loops represent Class-1 and Class-2B operations. Physical implementation of (d) NOT, (e) reversible NAND, and (f) reversible OR gates.

system. For example, NOT can be represented by a onedimensional Boolean line where the node at its two edges represents the two-input state of 0 and 1 , whereas the output state is denoted by empty and filled nodes for 0 and 1 output states, respectively, i.e.,

$$
{ }_{\square \longrightarrow \square}^{(0)}(1)
$$

which can be located at the lower quadrant of VPDs in Fig. 6(a). The physical implementation of NOT can be determined as follows: the Boolean line is horizontally aligned along a constant level of negative $V_{G 2}$ and the switching of $V_{G 1}$ from zero to positive value changes the conductance from $D+$ block to "OFF" state. Correspondingly, by fixing gate $G_{2}$ at a negative reference voltage and feeding the input signal into gate $G_{1}$, NOT operation is obtained [see Fig. 6(d)].

We now employ this graphical method to extract the permissible two-input Boolean operations, represented by Boolean loops, from the VPDs. We use the designation of "Class $X$ " to catalog all 16 types of Boolean logical operations where $X=0,1,2,3,4$ denotes the number of filled nodes in the Boolean loop. We first define Class- 0 and Class-4 logic where the four nodes at the vertices are either all empty or all filled. This corresponds to the trivial operations of "always-ON" and "always-OFF":

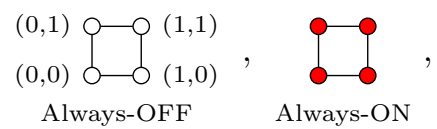

which can be implemented by drawing a Boolean loop lying completely outside and inside the $D_{ \pm}$conductance blocks, respectively. Note that the input address $(A, B)$ is explicitly marked in the always-OFF Boolean loop and is omitted in the following discussion for simplicity.
Class-1 logics, in which the Boolean loop contains only one filled node, can be implemented via the large Boolean loops shown in the VPDs of $D+[$ Fig. 6(b)] and $D-$ [Fig. 6(c)] modes:

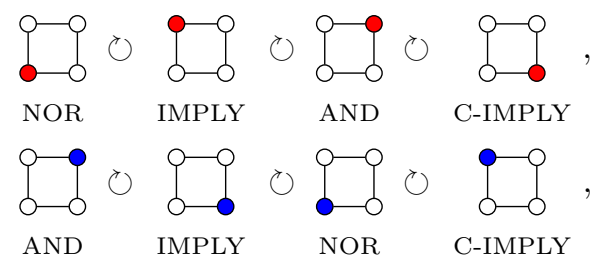

Importantly, the universal NOR gate falls into this class and can be implemented in $D+$ mode. Moreover, the exotic implication-type operations, such as the negations of implication (N-IMPLY) and of converse-implication (NC-IMPLY) can also be obtained via circular permutations of the Boolean loop in $V_{G 1}-V_{G 2}$ space [denoted by ' $\circlearrowright$ ' in Eq. (6)] and can be physically implemented by properly inverting and/or combining reference voltages with the voltage signals [see Figs. 6(d)-6(f) for examples].

The smaller Boolean loops shown in Figs. 6(b) and 6(c) represent Class-2A logics of two consecutive filled nodes:

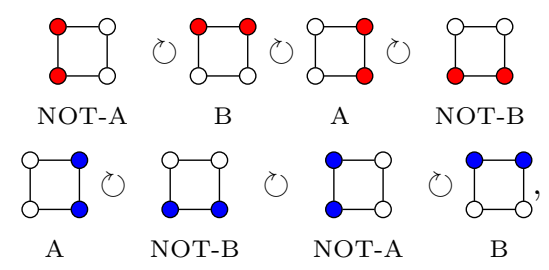

which are rather trivial logical operations. Class- $2 B$ logics, in which the two filled nodes are separated, provides more useful 
operations of XOR and XNOR. This class is obtainable from the larger Boolean loop shown in Fig. 6(a):

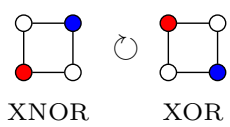

Interestingly, the two logical "ON" output states contain opposite valley polarizations as denoted by blue and red nodes. Such valley-color labeling manifests in a more remarkable way for Class-3 logics where three nodes are filled. This class is represented in the smaller Boolean loop in Fig. 6(a), i.e.,

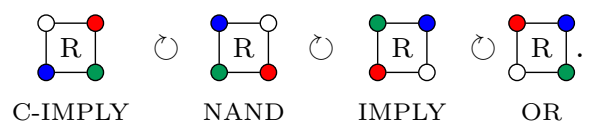

Equations (4)-(9) demonstrate that the valleytronic logic gates proposed here are capable of hosting all 16 types of two-input Boolean combinational logics. The Class-3 logics in Eq. (9) includes exotic implication (IMPLY), converse-implication (C-IMPLY) operations, and the conventional OR and NAND. NAND can be obtained via one circular permutation, i.e., by inverting one input and subsequently referencing it with a negative reference voltage as shown in Fig. 6(e). For OR gate, three circular permutations are required, which correspond to inverting the first input and referencing the second input with a negative voltage [Fig. 6(f)].

Class-3 logics are logically reversible as each of the three filled nodes is unambiguously labeled by $(2+1)$ valley polarization states. By reading out the valley polarization, any output can be unambiguously reverted back to their corresponding initial input. More remarkably, this reversible class of logics includes the all-important universal NAND gate. This reveals a remarkable potential of the valleytronic logical gate proposed here as a building block of classical reversible computer.

A general scheme of valleytronic-based reversible Boolean circuits is illustrated in Fig. 7(a). The three inputs (A, B, C) are computed reversibly into a final output $X_{2}$ in this example. A direct current $I_{0}$ is fed into each of the two-input valley gates and is modulated by the two inputs to yield an output current of a particular state of valley polarization. For valley gate \#1, A and B yield an intermediate output current $X_{1}$ of valley polarization $V_{1} . X_{1}$ is subsequently cascaded into valley gate $\# 2$ and its combination with the third input $C$ produces the final output current $X_{2}$ of valley polarization $V_{2}$. For a given $X_{2}$, the initial input states can be unambiguously recovered from the $V_{1}$ and $V_{2}$. Based on this general scheme, reversible half-adder (R-HA) can be implemented as shown in Fig. 7(b). From its truth table, it can be seen that the output state of " 1 " that corresponds to ambiguous input states of $(A, B)=$ $\{(1,0),(0,1)\}$ can be uniquely distinguishable from the valley polarization of the output [represented by $V$ in the truth table of Fig. 7(b)]. Two units of R-HA can be cascaded into a reversible full-adder (R-FA) [Fig. 7(c)]. In this case, the input states of $\left(C_{\mathrm{IN}}, A, B\right)=\{(1,0,0),(0,1,0),(0,0,1)\}$ can be unambiguously distinguished via the valley polarization $V$ of the intermediate $C$ output.

It should be emphasized that the valleytronic-based reversible circuit is fundamentally different from that of the
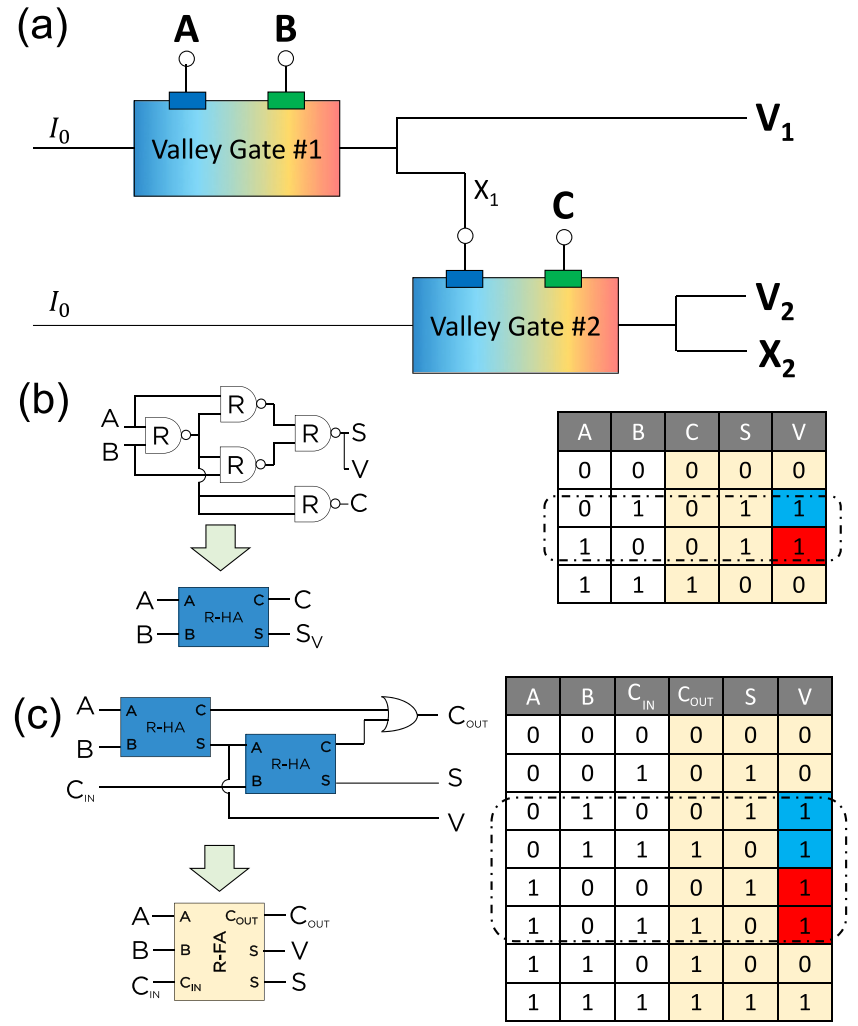

FIG. 7. Examples of valleytronic-based reversible Boolean circuit. (a) General scheme of a valleytronic-based reversible circuit. Three inputs (A, B, C) are operated by two gates to yield a final current output $X_{2}$. The input states can be unambiguously recovered via the two valley polarizations $V_{1}$ and $V_{2}$. (b) Reversible half-adder and (c) reversible full-adder with their truth table. Ambiguous operations are emphasized by dashed boxes. In these devices, the logical reversibility is established via the valley colors $V$.

traditional approach. Traditional reversible gates, such as Fredkin and Tofolli, achieve logical reversibility via multiple supplementary bits $[66,67]$ which inevitably introduce ancilla inputs and garbage outputs, bits unrelated to computation results and are only required for logical reversibility, into the circuits. In contrast, the reversible valleytronic logic gates proposed here harness the built-in valley degree of freedom for information storage. Extraction of information encoded in the valley is performed via an on-demand fashion, i.e., valley polarization is read out only when it is absolutely needed for logical reversibility. More importantly, the valleytronic reversible gate retains the conventional two-input format without involving any ancilla inputs. Thus, the combination of (i) reduced garbage outputs; (ii) complete absence of ancilla inputs; and (iii) the retaining of conventional two-input format suggests that the valleytronic approach for reversible computation can potentially be more advantageous than the traditional approach. We further note that although logical reversibility can break the Landauer's limit, it does not warrant the full elimination of energy dissipation. Energy dissipation in a reversible computer shall remain finite due to the inevitable physicalirreversibility of electronic devices and circuits [72,73]. Finally, we remark that it remains a technological challenge to fabricate the proposed valleytronic devices at current stage. 
The search for an ideal merging Dirac cone condensed matter system shall form an ongoing task before the full potential of valleytronic-based logic gate architecture proposed here can be tapped.

\section{CONCLUSION}

In summary, we study the pseudospin-assisted valleycontrasting quantum tunneling in $2 \mathrm{MDS}$ and show that such effect can be harnessed to create valley filter, valve, and logic gate. These valleytronic devices exhibit multiple unusual characteristics including (i) all-electric controllable; (ii) stable valley polarization that persists over semi-infinite gate voltage windows; (iii) colossal VPMR effect of well over $10000 \%$; (iv) flexibility to be permuted into any two-input Boolean gates; and (v) capable of performing reversible Boolean classical computation with reduced garbage and total absence of ancilla bits. The union of valley degree of freedom and digital computing offers an exciting solidstate platform for valleytronic-based information processing and for reversible computing which is ultimately required to lower hardware power consumption beyond the bound of Landauer's limit [74]. As logical reversibility is a prerequisite for quantum gate [75], we anticipate the universal reversible valleytronic logic gates proposed here to play a role in quantum [76] and quantum-classical hybrid computers [77].

\section{ACKNOWLEDGMENTS}

We thank K. J. A. Ooi, M. Zubair, and S.-H. Tan for helpful discussion. This work is funded by A*STAR AME IRG (Grant No. A1783c0011) and Singapore Ministry of Education MOETier-2 Grant (Grant No. T2MOE1401).

\section{APPENDIX A: CHIRALITY OF THE MERGING DIRAC CONES}

In this appendix, we show that the two Dirac split cones predicted by the universal Hamiltonian [Eq. (1) of main text] for $\Delta<0$ contain opposite chirality. We first expand the Hamiltonian around the two Dirac points, i.e., $\mathbf{k} \rightarrow \tilde{\mathbf{k}}$ where $\tilde{\mathbf{k}}=\left(\mathcal{D} \sqrt{|\Delta|}+\delta k_{x}, \delta k_{y}\right), \delta \mathbf{k} \equiv\left(\delta k_{x}, \delta k_{y}\right)$ is a small shift of wave vector and $\mathcal{D}= \pm 1$ denotes the two Dirac cones. By keeping terms first order in $\left(\delta k_{x}, \delta k_{y}\right)$, the dimensionless Hamiltonian becomes can be decoupled for each of the Dirac cone, i.e.,

$$
\hat{\mathcal{H}}_{\delta \mathbf{k}}^{(\mathcal{D})}=\left(\begin{array}{cc}
0 & \mathcal{D} \sqrt{|\Delta|} \delta k_{x}-i \delta k_{y} \\
\mathcal{D} \sqrt{|\Delta|} \delta k_{x}+i \delta k_{y} & 0
\end{array}\right)
$$

which coincides with the gapless Dirac Hamiltonian of graphene except that the "Fermi velocity" is anisotropic between $\delta k_{x}$ and $\delta k_{y}$ directions. The chirality operators can be defined as $\hat{\chi}_{\mathcal{D}}=\left(\overrightarrow{\mathcal{K}}_{\mathcal{D}} /\left|\overrightarrow{\mathcal{K}}_{\mathcal{D}}\right|\right) \cdot \hat{\sigma}$ where $\overrightarrow{\mathcal{K}}_{\mathcal{D}} \equiv\left(\mathcal{D} \sqrt{|\Delta|} \delta k_{x}, \delta k_{y}\right)$ is an "anisotropic wave vector." By defining a phase factor as $\phi_{\delta \mathbf{k}} \equiv \tan ^{-1}\left(\delta k_{y} / \sqrt{|\Delta|} \delta k_{x}\right)$, the eigenenergy and eigenstate can be solved, respectively, as $\varepsilon_{\delta \mathbf{k}, s}=s\left|\overrightarrow{\mathcal{K}}_{\mathcal{D}}\right|$ and $\left|\psi_{\delta \mathbf{k}, \mathcal{D}}\right\rangle=$ $\left(1, s e^{i \mathcal{D} \phi_{\delta \mathbf{k}}}\right)^{\mathcal{T}} e^{i \delta \mathbf{k} \cdot \mathbf{r}}$, where $\mathbf{r} \equiv(x, y)$ and $s= \pm 1$ is the band index. $\hat{\chi}_{\mathcal{D}}$ commutes with $\hat{\mathcal{H}}_{\delta \mathbf{k}}^{(\mathcal{D})}$ and follows the eigenvalue equation: $\hat{\chi}_{\mathcal{D}}\left|\psi_{\delta \mathbf{k}, \mathcal{D}}\right\rangle=\chi_{\mathcal{D}}\left|\psi_{\delta \mathbf{k}, \mathcal{D}}\right\rangle$, where the chirality eigen- value is $\chi_{\mathcal{D}}=s \mathcal{D}$. This demonstrates that $\chi_{\mathcal{D}}$ has opposite sign between $\mathcal{D}= \pm 1$ valleys.

\section{APPENDIX B: DERIVATION OF TUNNELING CONDUCTANCE OF CHIRAL VALLEY FILTER}

We consider quantum tunneling across a $1 \mathrm{D}$ square potential along the $x$ direction:

$$
U(x)=V_{g}[\Theta(x)-\Theta(x-d)]
$$

where $V_{g} \equiv e \mathcal{V}_{g} / \varepsilon_{0}$ is a dimensionless potential barrier/well height determined by the gate voltage $\mathcal{V}_{g}$ and $d=k_{0} d_{0}$ is the dimensionless width parameter that corresponds to the barrier width $d_{0}$. By replacing $k_{x} \rightarrow-i \partial / \partial_{x}$, the Schrödinger can be written explicitly as

$$
\begin{aligned}
& \left(\begin{array}{cc}
0 & -\frac{\partial^{2}}{\partial x^{2}}+\Delta-i k_{y} \\
-\frac{\partial^{2}}{\partial x^{2}}+\Delta+i k_{y}
\end{array}\right) \psi(x) \\
& =\left[\varepsilon_{k}-U(x)\right] \psi(x)
\end{aligned}
$$

which can be decoupled as

$$
\left(\frac{\partial^{4}}{\partial x^{4}}-2 \Delta^{2} \frac{\partial^{2}}{\partial x^{2}}\right) \phi_{A, B}=\left(\left[\varepsilon_{k}-U(x)\right]^{2}-k_{y}^{2}-\Delta^{2}\right) \phi_{A, B},
$$

where the $\psi(x)=\left(\phi_{A}, \phi_{B}\right)^{\mathcal{T}}$ is the pseudospinor wavefunction. The solutions of the first pseudospinor component with $U(x)=0$ can be solved as $\phi_{A}^{(\lambda \eta)}=\exp \left(i k_{x}^{(\lambda \eta)} x\right)$, where $\lambda=$ $\pm 1, \eta= \pm 1$,

$$
k_{x}^{(\lambda \eta)}=\lambda \sqrt{\eta\left(\varepsilon_{k}^{2}-k_{y}^{2}\right)^{1 / 2}-\Delta}
$$

and the energy eigenvalue is given as

$$
\varepsilon_{\mathbf{k}}= \pm \sqrt{\left(k_{x}^{(\lambda \eta) 2}+\Delta\right)^{2}+k_{y}^{2}}
$$

For $\Delta<0$ and $\left(\varepsilon_{\mathbf{k}}^{2}-k_{y}^{2}\right)<|\Delta|$, all eigenstates are propagating states with purely real $k_{x}$. The corresponding group velocity is given as $v_{x}^{(\lambda \eta)}=\partial \varepsilon_{\mathbf{k}} / \partial k_{x}^{(\lambda \eta)}=\lambda \eta\left(\varepsilon_{\mathbf{k}}^{2}-k_{y}^{2}\right)^{1 / 2} / \varepsilon_{\mathbf{k}}$ By comparing $v_{x}^{(\lambda \eta)}$ with $k_{x}^{(\lambda \eta)}$, the eigenstate can be determined as electronlike (holelike) if the product $\left(\lambda \eta / \varepsilon_{\mathbf{k}}\right)$ has the same (opposite) sign as $\lambda$ which signifies group velocity being (anti)parallel with the wave vector. The group velocity and the electron/hole nature of the $(\lambda, \eta)$ branches are shown in Fig. 8. This corresponds to the low-energy regime in which the energy dispersion splits into two distinct Dirac cones. For $\left(\varepsilon_{\mathbf{k}}^{2}-k_{y}^{2}\right)>|\Delta|$, the two branches of $\eta=-1$ merge and the corresponding eigenstate becomes evanescent due to the merging of Dirac cones.

The second pseudospinor component can be solved as

$$
\phi_{B}^{(\lambda \eta)}=\frac{\eta \sqrt{\varepsilon_{k}^{2}-k_{y}^{2}}+i k_{y}}{\varepsilon_{k}} e^{i k_{x}^{(\lambda \eta)} x} .
$$




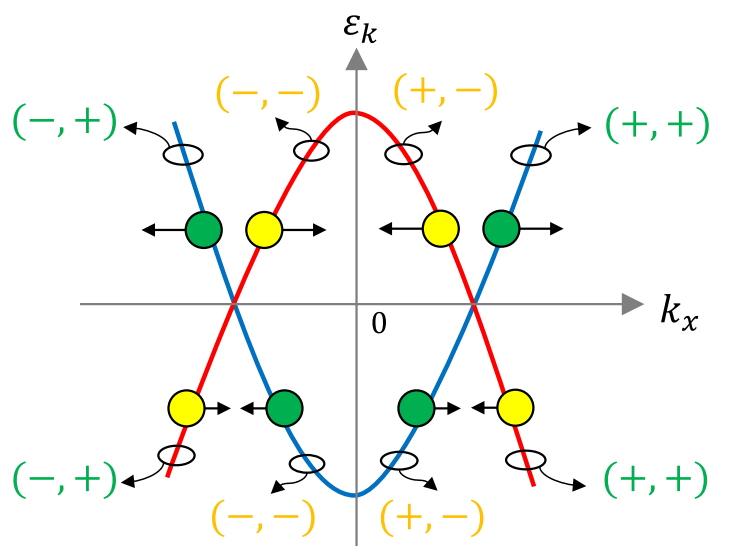

FIG. 8. Energy dispersion of merging Dirac cone system at $k_{y}=$ 0 . The $(\lambda, \eta)$ index of various branches is marked. The arrows denote the direction of the group velocity $v_{x}^{(\lambda \eta)}$. Electronlike $\left(v_{x}^{(\lambda \eta)}\right.$ parallel with $k_{x}$ ) and holelike $\left(v_{x}^{(\lambda \eta)}\right.$ antiparallel with $k_{x}$ ) quasiparticles are denoted by green and yellow circles, respectively.

Correspondingly, the normalized eigenstate outside the barrier and that inside the barrier are given, respectively, as

$$
\begin{aligned}
& \psi^{(\lambda \eta)}(x)=\frac{1}{\sqrt{2}}\left(\frac{1}{\eta \sqrt{\varepsilon_{\mathbf{k}}^{2}-k_{y}^{2}}+i k_{y}}\right)^{i k_{\mathbf{k}}^{(\lambda \eta)} x}, \\
& \tilde{\psi}^{(\lambda \eta)}(x)=\frac{1}{\sqrt{2}}\left(\frac{\eta \sqrt{\varepsilon_{\mathbf{k}}^{2}-k_{y}^{2}}+i k_{y}}{\varepsilon_{\mathbf{k}}-V_{g}}\right) e^{i q_{x}^{(\lambda \eta)} x},
\end{aligned}
$$

where $q_{x}^{\lambda \eta}=\lambda \sqrt{\eta\left[\left(\varepsilon_{k}-U_{0}\right)^{2}-k_{y}^{2}\right]^{1 / 2}-\Delta}$. The pseudospin vector $\mathbf{S}=\left(S_{x}, S_{y}\right)$ can be determined as

$$
\mathbf{S}=\psi^{(\lambda \eta) \dagger} \boldsymbol{\sigma} \psi^{(\lambda \eta)}=\left(\frac{k_{x}^{2}+\Delta}{\varepsilon_{\mathbf{k}}}, \frac{k_{y}}{\varepsilon_{\mathbf{k}}}\right) .
$$

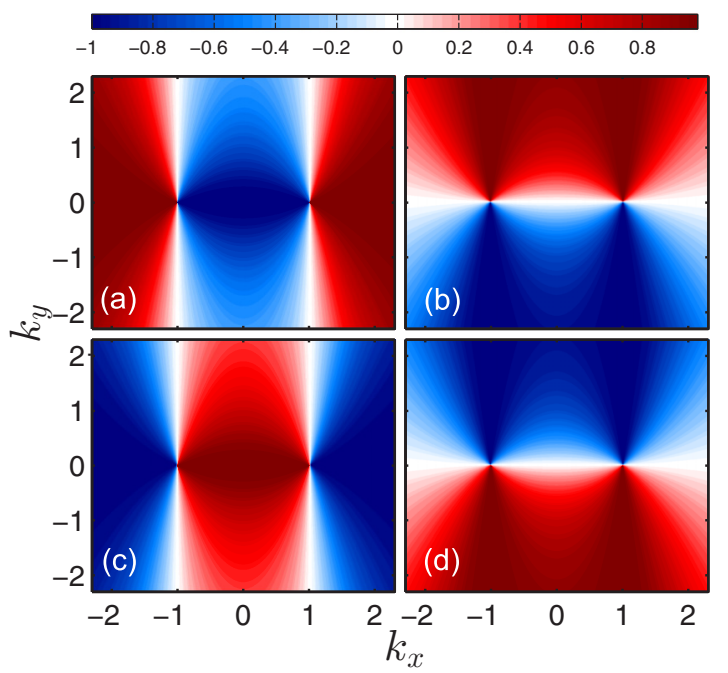

FIG. 9. Pseudospin texture of a merging Dirac cone system. (a), (b) Shows, respectively, the $x$-component pseudospin and $y$ component pseudospin textures of a merging Dirac cone system for $\varepsilon_{\mathbf{k}}<0$. (c), (d) Same as (a) and (b) but for $\varepsilon_{\mathbf{k}}>0$.
In Fig. 9, the $x$ and $y$ components of the pseudospin are plotted with $\Delta=-1$. At the vicinity of the Dirac points $\mathbf{k}=( \pm \sqrt{|\Delta|}, 0), S_{x}$ and $S_{y}$ behave in a contrasting way. The $S_{y}$ component is identical for both valleys while the $S_{x}$ component exhibits a sign change between the two valleys. Thus, $D+$ and $D$ - valleys are indistinguishable for $k_{y}$-directional transport while a strong valley contrast manifests in the $k_{x}$-directional transport and the two valleys have opposite chirality.

The total wave functions in regions I, II, and III are given as

$$
\begin{aligned}
\Psi_{\mathrm{I}}(x) & =\psi^{(\mathcal{D D})}(x)+\sum_{\eta= \pm} r_{\mathcal{D}}^{(\eta)} \psi^{(-\eta \eta)}(x) \\
\Psi_{\mathrm{II}}(x) & =\sum_{\eta= \pm} a^{(-\eta \eta)} \tilde{\psi}^{(-\eta \eta)}(x)+b^{(\eta \eta)} \tilde{\psi}^{(\eta \eta)}(x) \\
\Psi_{\mathrm{III}}(x) & =\sum_{\eta= \pm} t_{\mathcal{D}}^{(\eta)} \psi^{(\eta \eta)}(x)
\end{aligned}
$$

where $t_{\mathcal{D}}^{\left(\mathcal{D}^{\prime}\right)}$ and $r_{\mathcal{D}}^{\left(\mathcal{D}^{\prime}\right)}$ are the transmission and reflection, respectively. The index $\mathcal{D}, \mathcal{D}^{\prime}= \pm 1$ denotes the two valleys. The transmission and reflection coefficients can be solved by matching $\Psi_{\mathrm{I}}, \Psi_{\mathrm{II}}$, and $\Psi_{\mathrm{III}}$ at the boundaries of $x=0$ and $d$ via

$$
\begin{array}{r}
\Psi_{\mathrm{I}}(x=0)=\Psi_{\mathrm{II}}(x=0), \\
\Psi_{\mathrm{II}}(x=d)=\Psi_{\mathrm{III}}(x=d)
\end{array}
$$

and

$$
\begin{aligned}
& \left.\frac{d \Psi_{\mathrm{I}}(x)}{d x}\right|_{x=0}=\left.\frac{d \Psi_{\mathrm{II}}(x)}{d x}\right|_{x=0}, \\
& \left.\frac{d \Psi_{\mathrm{II}}(x)}{d x}\right|_{x=d}=\left.\frac{d \Psi_{\mathrm{III}}(x)}{d x}\right|_{x=d} .
\end{aligned}
$$

This forms a system of equations given as

$$
\begin{aligned}
& \left(\begin{array}{llll}
\tilde{\mathbb{K}}(0) & -\tilde{\mathbb{Q}}(0) & -\mathbb{Q}(0) & \mathbf{O}_{4 \times 2} \\
\mathbf{O}_{4 \times 2} & -\tilde{\mathbb{Q}}(d) & -\mathbb{Q}(d) & \mathbb{K}(d)
\end{array}\right)\left(\begin{array}{l}
\mathbf{R}^{(\mathcal{D})} \\
\mathbf{A}^{(\mathcal{D})} \\
\mathbf{B}^{(\mathcal{D})} \\
\mathbf{T}^{(\mathcal{D})}
\end{array}\right) \\
& =\left(\begin{array}{c}
\left.-\mathcal{K}^{(\mathcal{D})} 0\right) \\
\mathbf{O}_{4 \times 1}
\end{array}\right),
\end{aligned}
$$

where $\mathbf{O}_{M \times N}$ is a $M \times N$ zero matrix, the $4 \times 2$ matrices $\mathbb{K}$, $\tilde{\mathbb{K}}, \mathbb{Q}$, and $\tilde{\mathbb{Q}}$, are defined as

$$
\begin{aligned}
& \mathbb{K}(x) \equiv e\left(\begin{array}{cc}
\psi^{(++)}(x) & \psi^{(--)}(x) \\
k_{x}^{(++)} \psi^{(++)}(x) & k_{x}^{(--)} \psi^{(--)}(x)
\end{array}\right), \\
& \tilde{\mathbb{K}}(x) \equiv\left(\begin{array}{cc}
\psi^{(-+)}(x) & \psi^{(+-)}(x) \\
k_{x}^{(-+)} \psi^{(-+)}(x) & k_{x}^{(+-)} \psi^{(+-)}(x)
\end{array}\right), \\
& \mathbb{Q}(x) \equiv\left(\begin{array}{cc}
\tilde{\psi}^{(-+)}(x) & \psi^{(+-)}(x) \\
q_{x}^{(-+)} \tilde{\psi}^{(-+)}(x) & q_{x}^{(+-)} \tilde{\psi}^{(+-)}(x)
\end{array}\right), \\
& \tilde{\mathbb{Q}}(x) \equiv\left(\begin{array}{cc}
\tilde{\psi}^{(++)}(x) & \tilde{\psi}^{(--)}(x) \\
q_{x}^{(++)} \tilde{\psi}^{(++)}(x) & q_{x}^{(--)} \tilde{\psi}^{(--)}(x)
\end{array}\right)
\end{aligned}
$$

and $\mathcal{K}^{(\mathcal{D})}(x) \equiv\left(\psi^{(\mathcal{D D})}(x), k_{x}^{(\mathcal{D D}))} \psi^{(\mathcal{D D})}(x)\right)^{\mathcal{T}}$ with $\mathcal{D}= \pm 1$ indicates the valley index of the incident electron. The transport 
coefficients are compactly contained in

$$
\begin{aligned}
& \mathbf{R}^{(\mathcal{D})}=\left(\begin{array}{l}
r_{\mathcal{D}}^{(\mathcal{D})} \\
r_{-\mathcal{D}}^{(\mathcal{D})}
\end{array}\right), \quad \mathbf{T}^{(\mathcal{D})}=\left(\begin{array}{c}
t_{\mathcal{D}}^{(\mathcal{D})} \\
t_{-\mathcal{D}}^{(\mathcal{D})}
\end{array}\right), \\
& \mathbf{A}^{(\mathcal{D})}=\left(\begin{array}{c}
a_{\mathcal{D}}^{(\mathcal{D})} \\
a_{-\mathcal{D}}^{(\mathcal{D})}
\end{array}\right), \quad \mathbf{B}^{(\mathcal{D})}=\left(\begin{array}{c}
b_{\mathcal{D}}^{(\mathcal{D})} \\
b_{-\mathcal{D}}^{(\mathcal{D})}
\end{array}\right) .
\end{aligned}
$$

Finally, the probability current conservation can be written as

$$
1=\sum_{\mathcal{D}^{\prime}= \pm}\left(-\frac{v_{x}^{\left(-\mathcal{D}^{\prime} \mathcal{D}^{\prime}\right)}}{v_{x}^{(\mathcal{D D})}}\left|r_{\mathcal{D}^{\prime}}^{(\mathcal{D})}\right|^{2}+\frac{v_{x}^{\left(\mathcal{D}^{\prime} \mathcal{D}^{\prime}\right)}}{v_{x}^{(\mathcal{D D})}}\left|t_{\mathcal{D}^{\prime}}^{(\mathcal{D})}\right|^{2}\right),
$$

where the velocity expectation value is defined as

$$
v_{x}^{\left(u u^{\prime}\right)} \equiv \psi^{\left(u u^{\prime}\right) \dagger} \frac{\partial \hat{\mathcal{H}}_{\mathbf{k}}}{\partial k_{x}} \psi^{\left(u u^{\prime}\right)} .
$$

The reflection and tunneling probabilities can then be solved as

$$
R_{\mathcal{D}^{\prime}}^{(\mathcal{D})}=-\frac{v_{x}^{\left(-\mathcal{D}^{\prime} \mathcal{D}^{\prime}\right)}}{v_{x}^{(\mathcal{D D})}}\left|r_{\mathcal{D}^{\prime}}^{(\mathcal{D})}\right|^{2}, \quad T_{\mathcal{D}^{\prime}}^{(\mathcal{D})}=\frac{v_{x}^{\left(\mathcal{D}^{\prime} \mathcal{D}^{\prime}\right)}}{v_{x}^{(\mathcal{D D})}}\left|t_{\mathcal{D}^{\prime}}^{(\mathcal{D})}\right|^{2} .
$$

The $\mathcal{D}$-polarized ballistic tunneling current, under bias voltage $V_{B}$, is given as

$$
\begin{aligned}
\mathcal{J}^{(\mathcal{D})}\left(V_{B}, T\right)= & \frac{4 e \varepsilon_{0} k_{0}}{(2 \pi)^{2} \hbar} \int d k_{x} d k_{y}\left(\frac{\partial \varepsilon_{\mathbf{k}}}{\partial k_{x}}\right) \\
& \times T\left(k_{y}, \varepsilon_{\mathbf{k}}, V_{g}\right) f\left(\varepsilon_{\mathbf{k}}, T\right),
\end{aligned}
$$

where $f\left(\varepsilon_{\mathbf{k}}, T\right)$ is the Fermi-Dirac distribution function. At low temperature and small bias voltage, $f\left(\varepsilon_{\mathbf{k}}, T\right) \rightarrow$ $\left(e V / \varepsilon_{0}\right) \delta\left(\varepsilon_{\mathbf{k}}-\varepsilon_{F}\right)$. The ballistic conductance $G^{(\mathcal{D})}=$ $\mathcal{J}^{(\mathcal{D})} / V_{B}$ becomes

$$
G^{(\mathcal{D})}\left(\varepsilon_{F}, V_{g}\right)=G_{0} \int d k_{y} T\left(k_{y}, \varepsilon_{F}, V_{g}\right),
$$

where $G_{0} \equiv W g_{0} k_{0} / 2 \pi$ and $g_{0} \equiv 4 e^{2} / h$.

Before closing this section, we briefly discuss the intervalley scattering probabilities $T_{\mp}^{( \pm)}$, which follow the following symmetry:

$$
T_{\mp}^{( \pm)}\left(\varepsilon_{\mathbf{k}}, V_{g}\right)=T_{ \pm}^{(\mp)}\left(\varepsilon_{\mathbf{k}}, V_{g}\right)
$$

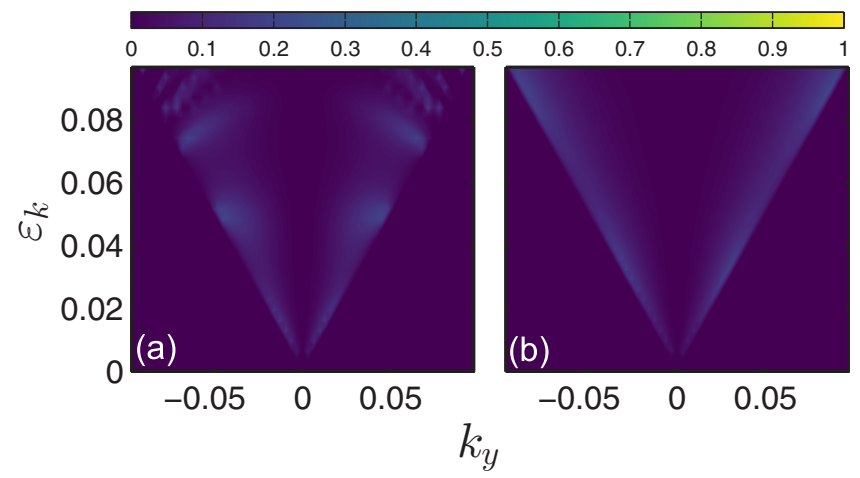

FIG. 10. Intervalley transmission probabilities $T_{\mp}^{( \pm)}$, plotted with (a) $V_{g}=0.2$ and (b) $V_{g}=-0.2(d=75, \Delta=0.1)$. Note that $T_{\mp}^{( \pm)}=T_{ \pm}^{(\mp)}$.

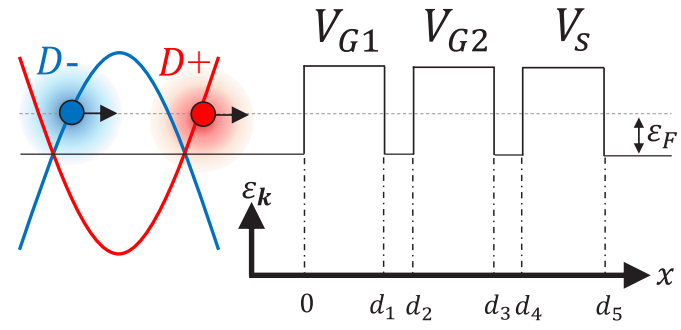

FIG. 11. Tunneling band diagram of valley valve device.

The numerical results of $T_{\mp}^{( \pm)}$are shown in Fig. 10. It can be seen that due to the mismatching of pseudospin, intervalley scattering is negligibly small and is only slightly raised at large $\left|k_{y}\right|$ due to the presence of a narrow "strip" of forward propagation branch at large $\left|k_{y}\right|$ that matches the chirality of opposing valleys [see pseudospin winding configuration in Figs. 3(b) and 3(c)].

\section{APPENDIX C: MODELING OF TUNNELING CONDUCTANCE IN A VALLEY VALVE}

The chiral valley valve is modeled using the following potential profile (see Fig. 11 for the tunneling structure):

$$
U(x)=\left\{\begin{array}{l}
V_{G 1}, 0<x<d_{1} \\
V_{G 2}, d_{2}<x<d_{3} \\
V_{s}, d_{4}<x<d_{5} \\
0, \text { otherwise }
\end{array}\right.
$$

where $V_{G 1,2} \equiv e \mathcal{V}_{G 1,2} / \varepsilon$ represents the dimensionless form of the first and second gate voltage $\mathcal{V}_{G 1,2}$, and $V_{s} \equiv e \mathcal{V}_{s} / \varepsilon_{0}$ represents the that of the selector voltage $\mathcal{V}_{s}$. The barrier widths corresponding to these gates are $d_{1},\left(d_{3}-d_{2}\right)$, and $\left(d_{5}-d_{4}\right)$, respectively. Similarly, the transport coefficients can be derived by matching the wave functions at each boundary, i.e., $x=$ $0, d_{i}$ (where $i=1,2,3,4,5$ ) via Eqs. (B10) and (B11). This leads to a system of 24 equations which are numerically solved to obtain the transmission coefficients. The corresponding transport probabilities and valley-polarized conductance are calculated via Eqs. (B17) and (B19), respectively.

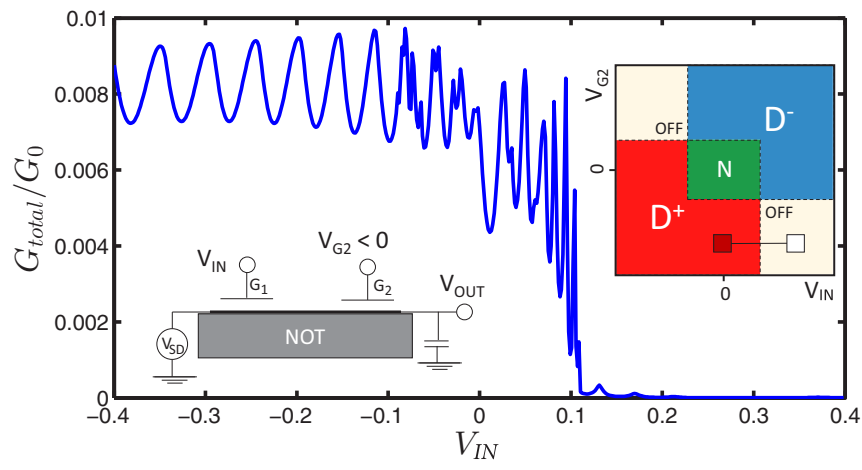

FIG. 12. Conductance-voltage characteristic of NOT gate with a fixed $V_{G 2}=0.2$. Insets show the Boolean line of NOT operation in the VPD with $V_{s}=0$, and the gate configurations. 


\section{APPENDIX D: SWITCHING CHARACTERISTICS OF VALLEYTRONIC-BASED NOT-GATE}

As a proof of concept, we explicitly show the conductancevoltage characteristics of a valleytronic-based NOT gate in Fig. 12. The total conductance as a function of input voltage $V_{\mathrm{IN}}$ is calculated as $G_{\text {total }}\left(V_{\mathrm{IN}}\right)=G_{+}\left(V_{\mathrm{IN}}\right)+G_{-}\left(V_{\mathrm{IN}}\right)$ and the $V_{G 2}=-0.2$ is a fixed reference voltage. The input signal is fed into the gate via $V_{\text {IN }}$. For $V_{\text {IN }} \leqslant 0$, the output total conductance is switched into a stable oscillation between $G_{\text {total }}\left(V_{\mathrm{IN}} \leqslant 0\right) \approx$
$0.007 G_{0}$ and $G_{\text {total }}\left(V_{\mathrm{IN}} \leqslant 0\right) \approx 0.009 G_{0}$ while for $V_{\mathrm{IN}} \geqslant 0.15$ the output conductance is switched off with $G_{\text {total }}$ in the order of $G_{\text {total }}\left(V_{\text {IN }} \geqslant 0.15\right) \approx 10^{-6} G_{0}$. This suggests that the implementation of NOT requires a minimum high/low input voltage difference of $\Delta V_{\mathrm{IN}} \approx 0.15$, which corresponds to a well-achievable value of approximately $150 \mathrm{meV}$. The switching time delay can be obtained via a full simulation that takes into account detail device geometry, and is beyond the scope of this work.
[1] A. Rycerz, J. Tworzydlo, and C. W. J. Beenakker, Nat. Phys. 3, 172 (2007).

[2] D. Xiao, W. Yao, and Q. Niu, Phys. Rev. Lett. 99, 236809 (2007).

[3] W. Yao, D. Xiao, and Q. Niu, Phys. Rev. B 77, 235406 (2008).

[4] Y. P. Shkolnikov, E. P. De Poortere, E. Tutuc, and M. Shayegan, Phys. Rev. Lett. 89, 226805 (2002).

[5] K. Takashina, Y. Ono, A. Fujiwara, Y. Takahashi, and Y. Hirayama, Phys. Rev. Lett. 96, 236801 (2006); K. Eng, R. N. McFarland, and B. E. Kane, ibid. 99, 016801 (2007); C. H. Yang, A. Rossi, R. Ruskov, N. S. Lai, F. A. Mohiyaddin, S. Lee, C. Tahan, G. Klimeck, A. Morello, and A. S. Dzurak, Nat. Commun. 4, 2069 (2013).

[6] Z. Zhu, A. Collaudin, B. Fauque, W. Kang, and K. Behnia, Nat. Phys. 8, 89 (2012); B. E. Feldman et al., Science 354, 316 (2016).

[7] J. Isberg, M. Gabrysch, J. Hammersberg, S. Majdi, K. K. Kovi, and D. J. Twitchen, Nat. Mater. 12, 760 (2013).

[8] A. Palyi and G. Burkard, Phys. Rev. Lett. 106, 086801 (2011).

[9] R. V. Gorbachev et al., Science 346, 448 (2014); M. Sui et al., Nat. Phys. 11, 1027 (2015); Y. Shimazaki, M. Yamamoto, I. V. Borzenets, K. Watanabe, T. Taniguchi, and S. Tarucha, ibid. 11, 1032 (2015).

[10] C. Zhang et al., Nat. Commun. 8, 13741 (2017).

[11] D. Xiao, G.-B. Liu, W. Feng, X. Xu, and W. Yao, Phys. Rev. Lett. 108, 196802 (2012); T. Cao et al., ibid. 3, 887 (2012); H. Zeng, J. Dai, W. Yao, D. Xiao, and X. Cui, Nat. Nanotechnol. 7, 490 (2012); K. F. Mak, K. He, J. Shan, and T. F. Heinz, ibid. 7, 494498 (2012).

[12] N. Rohling and G. Burkard, New J. Phys. 14, 083008 (2012); G. Y. Wu, N.-Y. Lue, and L. Chang, Phys. Rev. B 84, 195463 (2011); D. Culcer, A. L. Saraiva, B. Koiller, X. Hu, and S. Das Sarma, Phys. Rev. Lett. 108, 126804 (2012); E. A. Laird, F. Pei, and L. P. Kouwenhoven, Nat. Nanotechnol. 8, 565 (2013).

[13] H. Dery, P. Dalal, L. Cywinski1, and L. J. Sham, Nature (London) 447, 573 (2007); D. Loss and D. P. DiVincenzo, Phys. Rev. A 57, 120 (1998); B. Behin-Aein, D. Datta, S. Salahuddin, and S. Datta, Nat. Nanotechnol. 5, 266 (2010).

[14] J. L. O’Brien, A. Furusawa, and J. Vučković, Nat. Photonics 3, 687 (2009); H. Wei, Z. Wang, X. Tian, M. Kall, and H. Xu, Nat. Commun. 2, 387 (2011); Y. Fu, X. Hu, C. Lu, S. Yue, H. Yang, and Q. Gong, Nano Lett. 12, 5784 (2012); K. J. A. Ooi, H. S. Chu, P. Bai, and L. K. Ang, Opt. Lett. 39, 1629 (2014).

[15] International Roadmap Committee, International Technology Roadmap for Semiconductor 2.0: 2015 (Executive Report), 2013, http://www.itrs2.net/itrs-reports.html.
[16] D. S. L. Abergel and T. Chakraborty, Appl. Phys. Lett. 95, 062107 (2009).

[17] T. Fujita, M. B. A. Jalil, and S. G. Tan, Appl. Phys. Lett. 97, 043508 (2010).

[18] F. Zhai, X. Zhao, K. Chang, and H. Q. Xu, Phys. Rev. B 82, 115442 (2010).

[19] T. Low and F. Guinea, Nano Lett. 10, 3551 (2010).

[20] F. Zhai, Y. Ma, and K. Chang, New J. Phys. 13, 083029 (2011).

[21] Z. Wu, F. Zhai, F. M. Peeters, H. Q. Xu, and K. Chang, Phys. Rev. Lett. 106, 176802 (2011).

[22] F. Zhai, Nanoscale 4, 6527 (2012).

[23] F. Zhai and K. Chang, Phys. Rev. B 85, 155415 (2012).

[24] Y. Jiang, T. Low, K. Chang, M. I. Katsnelson, and F. Guinea, Phys. Rev. Lett. 110, 046601 (2013).

[25] T. Cai, S. A. Yang, X. Li, F. Zhang, J. Shi, W. Yao, and Q. Niu, Phys. Rev. B 88, 115140 (2013).

[26] L. Pratley and U. Zulicke, Appl. Phys. Lett. 104, 082401 (2014).

[27] M. M. Grujic, M. Z. Tadic, and F. M. Peeters, Phys. Rev. Lett. 113, 046601 (2014).

[28] L. S. Cavalcante, A. Chaves, D. R. da Costa, G. A. Farias, and F. M. Peeters, Phys. Rev. B 94, 075432 (2016).

[29] M. Settnes, S. R. Power, M. Brandbyge, and A.-P. Jauho, Phys. Rev. Lett. 117, 276801 (2016).

[30] M. Settnes, J. H. Garcia, and S. Roche, 2D Mater. 4, 031006 (2017).

[31] T. Sekera, C. Bruder, E. J. Mele, and R. P. Tiwari, Phys. Rev. B 95, 205431 (2017).

[32] M. M. Fogler, F. Guinea, and M. I. Katsnelson, Phys. Rev. Lett. 101, 226804 (2008).

[33] M. Ezawa, Phys. Rev. Lett. 109, 055502 (2012).

[34] F. Xu, Z. Yu, Y. Ren, B. Wang, Y. Wei, and Z. Qiao, New J. Phys. 18, 113011 (2016).

[35] I. Martin, Y. M. Blanter, and A. F. Morpurgo, Phys. Rev. Lett. 100, 036804 (2008).

[36] Z. Qiao, J. Jung, Q. Niu, and A. H. MacDonald, Nano Lett. 11, 3453 (2011).

[37] M. Ezawa 2012, New J. Phys. 14, 033003 (2012).

[38] F. Zhang, A. H. MacDonald, and E. J. Mele, Proc. Natl. Acad. Sci. USA 110, 10546 (2013).

[39] Z. Qiao, J. Jung, C. Lin, Y. Ren, A. H. MacDonald, and Q. Niu, Phys. Rev. Lett. 112, 206601 (2014).

[40] H. Pan, X. Li, F. Zhang, and S. A. Yang, Phys. Rev. B 92, 041404(R) (2015).

[41] J. R. Anglin and A. Schulz, Phys. Rev. B 95, 045430 (2017).

[42] L. Ju et al., Nature (London) 520, 650 (2015).

[43] J. Li et al., Nat. Nanotech. 11, 1060 (2016). 
[44] V. V. Cheianov, V. Fal'ko, and B. L. Altshuler, Science 315, 1252 (2007); G.-H. Lee, G.-H. Park, and Hu-Jong Lee, Nat. Phys. 11, 925 (2015); S. Chen, Z. Han, M. M. Elahi, K. M. M. Habib, L. Wang, B. Wen, Y. Gao, T. Taniguchi, K. Watanabe, J. Hone et al., Science 353, 1522 (2016).

[45] J. L. Garcia-Pomar, A. Cortijo, and M. Nieto-Vesperinas, Phys. Rev. Lett. 100, 236801 (2008); J. M. Pereira Jr., F. M. Peeters, R. N. Costa Filho, and G. A. Farias, J. Phys.: Condens. Matter. 21, 045301 (2009); Z. Wang and F. Liu, ACS Nano 4, 2459 (2010); D. Gunlycke and C. T. White, Phys. Rev. Lett. 106, 136806 (2011); Y. Liu, J. Song, Y. Li, Y. Liu, and Q.-F. Sun, Phys. Rev. B 87, 195445 (2013); S. P. Milovanovic and F. M. Peeters, Appl. Phys. Lett. 109 (2016); V. H. Nguyen, S. Dechamps, P. Dollfus, and J.-C. Charlier, Phys. Rev. Lett. 117, 247702 (2016).

[46] L. Tarruell, D. Greif, T. Uehlinger, G. Jotzu, and T. Esslinger, Nature (London) 483, 302 (2012).

[47] G. Montambaux, F. Piechon, J.-N. Fuchs, and M. O. Goerbig, Phys. Rev. B 80, 153412 (2009); Eur. Phys. J. B 72, 509 (2009); L.-K. Lim, J.-N. Fuchs, and G. Montambaux, Phys. Rev. Lett. 108, 175303 (2012).

[48] P. Delplace, Á. Gómez-León, and G. Platero, Phys. Rev. B 88, 245422 (2013); J.-S. Park and H. J. Choi, ibid. 92, 045402 (2015).

[49] J. Kim et al., Science 349, 723 (2015).

[50] S. S. Baik, K. S. Kim, Y. Yi, and H. J. Choi, Nano Lett. 15, 7788 (2015).

[51] T. Low, R. Roldán, H. Wang, F. Xia, P. Avouris, L. M. Moreno, and F. Guinea, Phys. Rev. Lett. 113, 106802 (2014); Y. Lu et al., NPJ Comp. Mater. 2, 16011 (2016)

[52] Q. Liu, X. Zhang, L. B. Abdalla, A. Fazzio, and A. Zunger, Nano Lett. 15, 1222 (2015); A. N. Rudenko, S. Yuan, and M. I. Katsnelson, Phys. Rev. B 92, 085419 (2015); S. Yuan, E. van Veen, M. I. Katsnelson, and R. Roldan, ibid. 93, 245433 (2016).

[53] B. Deng et al., Nat. Commun. 8, 14474 (2017).

[54] A. S. Rodin, A. Carvalho, and A. H. Castro Neto, Phys. Rev. Lett. 112, 176801 (2014); R. Fei, V. Tran, and L. Yang, Phys. Rev. B 91, 195319 (2015); Z. J. Xiang, G. J. Ye, C. Shang, B. Lei, N. Z. Wang, K. S. Yang, D. Y. Liu, F. B. Meng, X. G. Luo, L. J. Zou et al., Phys. Rev. Lett. 115, 186403 (2015); P.-L. Gong, D.-Y. Liu, K.-S. Yang, Z.-J. Xiang, X.-H. Chen, Z. Zeng, S.-Q. Shen, and L.-J. Zou, Phys. Rev. B 93, 195434 (2016); C.-H. Li, ibid. 95, 125417 (2017).

[55] C. Dutreix, E. A. Stepanov, and M. I. Katsnelson, Phys. Rev. B 93, 241404(R) (2016).

[56] S. M. Young, S. Zaheer, J. C. Y. Teo, C. L. Kane, E. J. Mele, and A. M. Rappe, Phys. Rev. Lett. 108, 140405 (2012); Z. Wang, Y. Sun, X.-Q. Chen, C. Franchini, G. Xu, H. Weng, X. Dai, and Z. Fang, Phys. Rev. B 85, 195320 (2012); Z. Wang, H. Weng, Q. Wu, X. Dai, and Z. Fang, ibid. 88, 125427 (2013); S. Jeon et al., Nat. Mater. 13, 851 (2014); H.-Z. Lu, S.-B. Zhang, and S.-Q. Shen, Phys. Rev. B 92, 045203 (2015); S. Y. Xu et al., Science 347, 294 (2015).
[57] M. Zhao, X. Zhang, and L. Li, Sci. Rep. 5, 16108 (2015); S. Zhang et al., Nano Lett. 17, 3434 (2017).

[58] J. Mathon and A. Umerski, Phys. Rev. B 63, 220403(R) (2001); T. Kimura, Y. Tomioka, H. Kuwahara, A. Asamitsu, M. Tamura, and Y. Tokura, Science 274, 1698 (1996); T. Kimura, A. Asamitsu, Y. Tomioka, and Y. Tokura, Phys. Rev. Lett. 79, 3720 (1997); S. S. P. Parkin, C. Kaiser, A. Panchula, P. M. Rice, B. Hughes, M. Samant, and S.-H. Yang, Nat. Mater. 3, 862 (2004).

[59] P. San-Jose, E. Prada, E. McCann, and H. Schomerus, Phys. Rev. Lett. 102, 247204 (2009); D. Pesin and A. H. MacDonald, Nat. Mater. 11, 409 (2012).

[60] M. Ye. Zhuravlev, R. F. Sabirianov, S. S. Jaswal, and E. Y. Tsymbal, Phys. Rev. Lett. 94, 246802 (2005); Y. W. Yin et al., Nat. Mater. 12, 397 (2013); L. Jiang et al., Nano Lett. 13, 5837 (2013).

[61] R. P. Feynman, Feynman Lectures on Computation (Westview Press, Boulder, CO, 2000).

[62] C. H. Bennett, IBM J. Res. Develop. 17, 525 (1973).

[63] M. I. Katsnelson, K. S. Novoselov, and A. K. Geim, Nat. Phys. 2, 620 (2006).

[64] M. Karnaugh, Trans. Am. Inst. Elect. Eng., Part I: Commun. Electron. 72, 593 (1953).

[65] R. Landauer, IBM J. Res. Develop. 5, 183 (1961); A. Berut, A. Arakelyan, A. Petrosyan, S. Ciliberto, R. Dillenschneider, and E. Lutz, Nature (London) 483, 187 (2012).

[66] T. Toffoli, in Automata, Languages and Programming, ICALP 1980, Lecture Notes in Computer Science, Vol. 85, edited by J. de Bakker and J. van Leeuwen (Springer, Berlin, Heidelberg, 1980), pp. 632-644.

[67] E. Fredkin and T. Toffoli, Int. J. Theor. Phys. 21, 219 (1982).

[68] J. Hardy and J. Shamir, Opt. Express 15, 150 (2007).

[69] R. P. Feynman, Opt. News 11, 11 (1985).

[70] From first-principles simulation of few-layer black phosphorus with merging Dirac cones [50], the average electron effective mass in $x$ direction is $m^{*} \approx 1.42 m_{e}$ where $m_{e}$ is the free-electron mass, and the $y$-direction Fermi velocity is $v_{F} \approx 2.8 \times 10^{5} \mathrm{~m} / \mathrm{s}$. Correspondingly, $\varepsilon \equiv 2 m^{*} v_{F}^{2} \approx 1.3 \mathrm{eV}$ and $k_{0} \equiv 2 m^{*} v_{F} / \hbar \approx$ $1.1 \mathrm{~nm}^{-1}$.

[71] R. Landauer, IBM J. Res. Develop. 1, 223 (1957).

[72] S. Lloyd, Nature (London) 406, 1047 (2000).

[73] I. L. Markov, Nature (London) 512, 147 (2014).

[74] M. Saeedi and I. L. Markov, ACM Comput. Surv. 45, 21 (2013).

[75] A. Barenco, C. H. Bennett, R. Cleve, D. P. DiVincenzo, N. Margolus, P. Shor, T. Sleator, J. A. Smolin, and H. Weinfurter, Phys. Rev. A 52, 3457 (1995).

[76] A. Steane, Rep. Prog. Phys. 61, 117 (1998).

[77] A. Peruzzo et al., Nat. Commun. 5, 4213 (2014); J. R. McClean, J. Romero, R. Babbush, and A. Aspuru-Guzik, New J. Phys. 18, 023023 (2016); S. Bravyi, G. Smith, and J. A. Smolin, Phys. Rev. X 6, 021043 (2016); B. Bauer, D. Wecker, A. J. Millis, M. B. Hastings, and M. Troyer, ibid. 6, 031045 (2016). 